\title{
Increased miR-21-3p in Injured Brain Microvascular Endothelial Cells after Traumatic Brain Injury Aggravates Blood-Brain Barrier Damage by Promoting Cellular Apoptosis and Inflammation through Targeting MAT2B
}

\author{
Xintong Ge,-4, ${ }^{1-\star}$ Wenzhu Li, ${ }^{4,5, *}$ Shan Huang,,5 Zhenyu Yin, ${ }^{4,5}$ Mengchen Yang, ${ }^{1-3}$ Zhenying Han, ${ }^{1-3}$ \\ Zhaoli Han, ${ }^{4,5}$ Fanglian Chen, ${ }^{2,3}$ Haichen Wang, ${ }^{6}$ Ping Lei, ${ }^{4,5}{ }^{*}$ and Jianning Zhang ${ }^{1-3, *}$
}

\begin{abstract}
Our recent articles have reported that increased miR-21-5p in brain after traumatic brain injury (TBI) could improve the neurological outcome through alleviating blood-brain barrier (BBB) damage. miR-21-3p is another mature miRNA derived from pre-miR-21 after Dicer Procession other than miR-21-5p. Its roles in various diseases, such as tumors and myocardial disease, aroused great interest for research in recent years. To further explore the function and underlying mechanism of miR-21, especially miR-21-3p, in regulating the pathological development of BBB damage after TBI, we designed this research and focused on studying the impact of miR-21-3p on apoptosis and inflammation in brain microvascular endothelial cells (BMVECs), the major cellular component of BBB. We performed controlled cortical impact on mouse brain and employed the oxygen glucose deprivation/reoxygenation (OGD)-treated bEnd.3 cells injury model. We found that the miR-21-3p level in BMVECs from injured cerebral cortex of controlled cortical impact (CCI) mice and bEnd.3 cells with OGD treatment were both increased after injury. For in vitro experiments, downregulation on the miR21-3p level by transfecting miR-21-3p antagomir in cultured cells alleviated OGD-induced BBB damage, characterized by decreased BBB leakage and increased expression of tight junction proteins. Besides, miR-21-3p antagomir could suppress cell death by anti-apoptosis and control inflammatory response by inhibiting the activity of NF- $\kappa \mathrm{B}$ signaling. Using luciferase reporter assay and a MAT2B-silenced shRNA vector, we further proved that miR-21-3p exerted the above functions through targeting MAT2B. In addition, in vivo experiments also confirmed that intracerebroventricular infusion of miR-21-3p antagomir could alleviate BBB leakage after TBI. It reduced Evans Blue extravasation and promoted the expression of tight junction proteins, thus contributed to improve the neurological outcome of CCI mice. Taken together, increased miR-21-3p in BMVECs after TBI was bad for restoration of injured BBB. Downregulation on the miR-21-3p level in injured brain could be a promising therapeutic strategy for BBB damage after TBI.
\end{abstract}

Keywords: apoptosis; blood-brain barrier; inflammation; miRNA; traumatic brain injury

\section{Introduction}

$\mathbf{T}$ RAUMATIC BRAIN INJURY (TBI) is a growing public health problem that has induced a high rate of death and longterm disability, especially in children and young adults. More than 50 million persons worldwide are affected annually by a new TBI case, with a global economic cost of approximately
\$US400 billion. ${ }^{1}$ With the acceleration of urbanization, the increase of traffic accidents, and the frequent occurrence of local wars, the incidence of TBI increases rapidly each year, which will make it the third leading cause of disease burden by $2020 .^{2}$

The pathology of TBI includes two general stages: primary brain damage and secondary brain damage. The primary damage is impact related and triggers acute pathological changes, such as

\footnotetext{
${ }^{1}$ Department of Neurosurgery, Tianjin Medical University General Hospital, Tianjin, China.

${ }^{2}$ Key Laboratory of Injuries, Variations and Regeneration of Nervous System, Tianjin Neurological Institute, Tianjin Medical University General Hospital, Tianjin, China.

${ }^{3}$ Key Laboratory of Post-Trauma Neuro-Repair and Regeneration in Central Nervous System, Ministry of Education, Tianjin, China.

${ }^{4}$ Laboratory of Neuro-Trauma and Neurodegenerative Disorders, Tianjin Geriatrics Institute, Tianjin Medical University General Hospital, Tianjin, China.

${ }^{5}$ Department of Geriatrics, Tianjin Medical University General Hospital, Tianjin, China.

${ }^{6}$ Department of Neurology, Duke University Medical Center, Durham, North Carolina.

*These authors contributed equally to this article.
} 
brain contusions, intracerebral hemorrhage, and shearing injuries. Parenchymal and vascular damage in the brain contributes to bloodbrain barrier (BBB) breakdown. The BBB is essential for maintaining cerebral homeostasis. It protects the brain from pathogenic microorganisms and other high molecular weight substances in the blood circulation in the physiological state. Its damage after TBI leads directly to secondary damages, including brain edema, local inflammatory response, and neuronal hyperexcitability, which begin hours to days after injury and can last for days to years.

These negative effects could induce a cascade of pathological processes, such as neovascularization, impaired cerebral blood flow, gliacytes dysfunction, and neurodegeneration. They progress, interact, and initiate severe complications, such as intracranial hypertension and brain hernia, which result in poor neurological prognosis, including death, coma, seizures, and cognitive/behavioral disabilities. ${ }^{3,4}$

For clinical treatment, the primary damage is considered to be untreatable for a short time window, whereas the "rolling" pathology of the following secondary damage allows an opportunity for intervention. Because BBB breakdown is the key pathological event that induces secondary damage, promoting the restoration of injured $\mathrm{BBB}$ for preventing or alleviating the breakdown-mediated secondary damage could be a promising treatment for those with TBI.

Currently, no readily available treatment has proven to be effective for preventing or reversing the BBB damage after TBI in the clinical setting. Corticosteroids, especially hydrocortisone and dexamethasone, are well-known agents with antiedematous properties acting on BBB. Since their introduction in the 1960s, they have been used widely in controlling brain edema after TBI. High-dose corticosteroids pulse therapy was thought to be effective at one time, whereas a clinical trial conducted in more than 40 countries (MRC CRSAH, 10,008 patients) suggested that the treatment had no therapeutic effect on patients with TBI. ${ }^{5}$ In addition, our recent research indicated that corticosteroids could aggravate neuronal injury in the hypothalamus after TBI, thus suppressing the stress response of the hypothalamic-pituitary-adrenal axis and inducing the critical illness-related corticosteroid insufficiency (CIRCI) that results in poor prognosis. ${ }^{6,7}$

Therapeutic hypothermia (also termed target temperature management) is a cornerstone in TBI management. Since the 1990s, scientists have found that mild reductions in brain temperature can reduce BBB disruption and improve histopathological and neurological outcomes in experimental animals with TBI. ${ }^{8,9}$ These results were confirmed by a number of single institutional clinical trials. Larger randomized multi-center trials, however, have failed to demonstrate the benefits of hypothermia therapy, suggesting that this therapy is limited by patient selection and administration time after TBI. ${ }^{10}$

Stem cell therapy, including transplantation of mesenchymal stromal cells (MSCs), ${ }^{11}$ multi-potent adult progenitor cells (MAPCs), ${ }^{12}$ and endothelial colony-forming cells (ECFCs) ${ }^{13}$ could restore BBB integrity and promote functional recovery after TBI (NCT02028104). Problems of ethics and biosafety have long been a concern, however, and limited its further development and clinical applications.

Molecular therapy is a new research hotspot in recent years. The representative drugs, erythropoietin and progesterone, have been reported widely to alleviate BBB damage in animal models, ${ }^{4,14}$ but phase III clinical trials failed to find their therapeutic effects in patients with TBI. ${ }^{15,16}$ Statins, such as atrovastatin (NCT02024373) and rosuvastatin (NCT00990028), which have a similar function in animals with TBI, are still in early clinical trials. ${ }^{17}$ Consequently, it is important to explore new therapeutic strategies for alleviating BBB damage after TBI in future study.

The microribonucleic acids (miRNAs) are a class of small noncoding RNAs that regulate gene expression at the post-transcriptional level and play vital roles in regulating pathological development in diseases. Our research group has engaged in studying the roles and mechanism of miRNAs in TBI since 2009. We have reported that the expression level of miR-21-5p in the brain was increased after TBI. ${ }^{18}$ It contributed to improving the neurological outcome of TBI by inhibiting neuronal apoptosis and promoting angiogenesis through targeting PTEN and activating Akt signaling in injured cerebral cortex and hippocampus. ${ }^{19,20}$ In addition, miR-21-5p in brain microvascular endothelial cells (BMVECs), the major cellular component of BBB, could alleviate BBB damage by promoting the expression of tight junction proteins via activating the angiopoietin-1/Tie-2 axis. ${ }^{21-23}$

miR-21-3p is another mature miRNA derived from pre-miR-21 after Dicer Procession other than miR-21-5p. Its roles in various diseases, such as tumors and myocardial disease, aroused great interest for research in recent years. ${ }^{24-26}$ As to TBI, previous studies reported that the miR-21-3p level in the injured brain was increased at $1 \mathrm{~h}, 6 \mathrm{~h}$, and $12 \mathrm{~h}$ post-injury using miRNA microarray and quantitative reverse transcription polymerase chain reaction (RT-PCR). ${ }^{27}$

To further explore the function and underlying mechanism of miR-21, especially miR-21-3p, in regulating BBB damage after TBI, we designed this research and focused on studying the impact of miR-21-3p on apoptosis and inflammation in injured BBB. The results could clarify the roles of miR-21-3p in regulating the pathological development of traumatic BBB damage, thus expanding the previous understanding of the function and mechanism of miR-21 in TBI. Our research is expected to open a new avenue of therapeutic strategies for TBI by manipulating the miR-21-3p level.

\section{Methods}

All experimental procedures were performed in accordance with the National Institutes of Health (NIH) Guide for the Care and Use of Laboratory Animals and approved by the Tianjin Medical University Animal Care and Use Committee (2017GHNS01T).

\section{Controlled cortical impact (CCI) mouse model}

Adult male C57BL/6 mice ( $\mathrm{n}=139$, aged 12 weeks, weighing 20$25 \mathrm{~g}$ ) were purchased from the Chinese Academy of Military Science (Beijing, China). Briefly, the mice were anesthetized with $10 \%$ chloride hydrate $(3.0 \mathrm{~mL} / \mathrm{kg})$ administered by intraperitoneal injection. They were then positioned in a stereotaxic device using ear bars.

After a midline scalp incision, a 3.0-mm craniotomy was performed centrally over the right parietal bone. The impounder tip of the injury device (eCCI model 6.3, American Instruments, Richmond, VA) was then extended to its full impact distance, positioned on the surface of the exposed dura mater, and reset to impact its surface. For moderateTBI induction, the impact parameters (velocity: $4.5 \mathrm{~m} / \mathrm{sec}$, depth: $2 \mathrm{~mm}$, dwell time: $200 \mathrm{msec}$ ) were set as reported. ${ }^{28}$ Any mice with a herniation of the dura mater were eliminated from the group.

After the impact, the craniotomy was closed with bone wax, and the scalp was sutured. The mice were placed in a well-ventilated cage at $37^{\circ} \mathrm{C}$ until they regained consciousness. Sham-operated mice underwent the same procedures except for the cortical impact. To determine the degree of brain injury, hematoxylin and eosin staining was performed on brain sections at $24 \mathrm{~h}$ post-CCI.

\section{Temporal profile of miR-21-3p level in BMVECs after $\mathrm{CCl}$}

The mice were sacrificed by transcardiac perfusion with cold phosphate buffered saline (PBS) at $3 \mathrm{~h}, 12 \mathrm{~h}, 24 \mathrm{~h}, 48 \mathrm{~h}, 72 \mathrm{~h}$, and 7 days post-CCI. The brains were dissected on ice, and the injured cerebral cortex was isolated immediately. To collect the BMVECs, ${ }^{29}$ the brain tissue was placed in $6 \mathrm{~mL} 30 \%$ dextran solution within $20 \mathrm{mM} \mathrm{NaHCO}_{3}, 10 \mathrm{mM}$ glucose, $1 \mathrm{mM}$ Na pyruvate, 
and $0.5 \%$ bovine serum albumin (BSA). The mixture was spun at $6000 \times g$ for $15 \mathrm{~min}$ at $4^{\circ} \mathrm{C}$. Next, the supernatant was removed, and the remaining pellet was passed through a $100-\mu \mathrm{m}$ nylon mesh (Becton Dickinson, Franklin Lakes, NJ). The filtrate was then passed through a 40- $\mu$ m nylon mesh, and the microvessels could be collected on the mesh. After a rinse by PBS, these samples were used for RT-PCR detection to quantify the miR-21-3p level in BMVECs.

\section{Cell culture and identification of mouse brain endothelial cell line (bEnd.3)}

The bEnd. 3 cells were cultured and maintained in the complete endothelial cell medium (ECM) with $10 \%$ fetal bovine serum (FBS) and $1 \%$ penicillin/streptomycin (ThermoFisher Scientific, Waltham, MA), and incubated at $37^{\circ} \mathrm{C}$ and $5 \% \mathrm{CO}_{2}$. Immunofluorescence staining of CD31 was performed for cell identification. Briefly, the cells were fixed in $4 \%$ paraformaldehyde for $15 \mathrm{~min}$ at room temperature, followed by treating with $5 \%$ BSA for $30 \mathrm{~min}$ at $37^{\circ} \mathrm{C}$ to block nonspecific staining. They were then incubated overnight at $4^{\circ} \mathrm{C}$ with anti-CD31 antibody. The next day, the cells were rinsed by PBS, and then incubated for $1 \mathrm{~h}$ at room temperature with the secondary antibody. The nuclei were counterstained with DAPI (Abcam).

\section{Oxygen glucose deprivation/reoxygenation (OGD) cell injury model}

For OGD treatment, the culture medium was replaced with the glucose-free endothelial cell medium (ECM). The cells were then incubated in an anaerobic chamber (HERACELL 150i, ThermoFisher Scientific) for $6 \mathrm{~h}$, in which the oxygen level was maintained below $0.5 \%$. After that, the glucose-free medium was replaced with normal growth medium, and the cells were once again incubated under normal culture conditions.

\section{Hopping probe ion conductance microscopy (HPICM) scanning on tight junctions}

HPICM was composed of a sample scan head SH01 (Ionscope, Melbourn, Cambridgeshire, UK) and an ICnano scanner controller (Ionscope). The SH01 with a nanopipette probe was placed on the inverted TiU microscope (Nikon, Tokyo, Japan). The ICnano controller managed two 100- $\mu \mathrm{m}$ PIHera piezo (P-621.2C, Physik Instrumente, Karlsruhe, Germany) to control cell movement in the horizontal $\mathrm{X}-\mathrm{Y}$ direction. The $\mathrm{Z}$ direction $25-\mu \mathrm{m}$ LISA piezo $(\mathrm{P}-$ 753.21C, Physik Instrumente) controlled the vertical positioning and hopping of the probe. In addition, an external Axon Multi Clamp700B amplifier (Molecular Devices, Sunnyvale, CA) provided a 200-mV DC voltage between the nanopipette electrode and the reference electrode, and monitored the ion current between the nanopipette tip and cell surface. When the hopping probe approached the cell surface, a $0.4 \%$ reduction of the reference DC current was set to maintain the probe away from the cell surface.

When HPICM scanning were performed, the nanopipettes that filled with Leibovitz's L15 medium (ThermoFisher Scientific) were pulled from the borosilicate glass (O.D. $1.00 \mathrm{~mm}$, I.D. $0.59 \mathrm{~mm}$, VitalSense Scientific Instruments, Wuhan, China) with a laser-based puller Model P-2000 (Sutter Instruments, Novato, CA). The time required to scan an area of $40 \mu \mathrm{m}^{2}$ with a typical resolution of $256 \times 256$ pixels was about $20 \mathrm{~min}$. The primary topography data were processed and analyzed using SICM Image Viewer software (Ionscope). ${ }^{30}$

\section{Temporal profile of miR-21-3p level in bEnd.3 cells after OGD treatment}

The cultured cells were detached and collected at $1 \mathrm{~h}, 3 \mathrm{~h}, 6 \mathrm{~h}$, $12 \mathrm{~h}, 24 \mathrm{~h}$, and $48 \mathrm{~h}$ post-OGD, and their miR-21-3p levels were detected by quantitative RT-PCR. The variation trend of miR-21$3 p$ expression in bEnd. 3 cells after OGD treatment was compared with that in BMVECs from injured cerebral cortex after CCI.

\section{Grouping methods and miR-21-3p oligomers transfection}

The cultured cells were divided randomly into four groups: normal cells (CON), OGD treatment (OGD), OGD + miR-21-3p antagomir (OGD +21-3p-), and OGD + antagomir negative control $(\mathrm{OGD}+\mathrm{NC})$. For miRNA transfection, miR-21-3p oligomers (sequences listed in Table 1; GenePharma, Shanghai, China) were diluted to a final concentration of $20 \mu \mathrm{M}$. Then 5- $\mu \mathrm{L}$ miRNA oligomers were combined with the same amount of lipofectamine-3000 (ThermoFisher Scientific) in 500- $\mu \mathrm{L}$ glucose-free ECM medium without FBS, and incubated for $20 \mathrm{~min}$ at room temperature to be the transfect solution. It was then added into the cultured cells for $6 \mathrm{~h}$ of transfection, followed by replacing with the complete ECM medium.

The OGD treatment was performed at $36 \mathrm{~h}$ post-transfection in the OGD +21-3p- group and the OGD + NC group. To evaluate the transfection efficiency, the FAM-labeled miR-21-3p antagomir was used for transfection. The FAM-positive cell that represents an effective transfection can be observed under the fluorescence microscope. At $6 \mathrm{~h}$ post-OGD $(48 \mathrm{~h}$ post-transfection, when the transfection efficacy reached the peak according to the manufacture's instructions), their numbers were counted in five randomly selected fields of a culture plate under $200 \times$ magnification, and the percentage of them in all cultured cells was calculated. Quantitative RT-PCR for miR-21-3p level after transfection and OGD treatment was also performed.

\section{Horseradish peroxidase (HRP) flux assay}

For this test, bEnd. 3 cells were seeded onto the upper chamber (UC) of Transwell Permeable Inserts $(0.4 \mu \mathrm{m}$ pore size, Corning, NY), where OGD treatment and cell transfection were performed. Then 2240- $\mu$ L complete ECM medium with 0.5-mg HRP (SigmaAldrich, St. Louis, MO) was added into the UC at $6 \mathrm{~h}$ post-OGD. To keep the fluid surface in the UC and the lower chamber (LC) in a same horizontal plane, the complete ECM medium was added into the LC. The $50-\mu \mathrm{L}$ culture medium of the $\mathrm{LC}$ was collected once an hour in the next $4 \mathrm{~h}$. These were then treated with the substrate of HRP for 3 min. The $50-\mu \mathrm{L} \mathrm{H}_{2} \mathrm{SO}_{4}(1 \mathrm{M})$ was used to stop the reaction. After that, the HRP contents were detected by a Microplate Reader (Varioskan Flash, Thermo Scientific) at the wavelength of $450 \mathrm{~nm}$. The concentration of HRP was measured according to the standard curve, and the final HRP flux rate was calculated as: [concentration $(\mathrm{LC}) \times$ Volume $(\mathrm{LC})] /[$ concentration $(\mathrm{UC}) \times$ Volume (UC) $] \times 100 \%$. $^{22}$

\section{In vitro tight junction proteins detection}

The bEnd. 3 cells were detached and collected at $6 \mathrm{~h}$ post-OGD. Western blotting for occludin, claudin-5 and ZO-1 was performed to quantify their expressions after treatment.

Table 1. Sequences of Mir-21-3P Oligomers

\begin{tabular}{lc} 
miR-21-3p oligomer & Sequences \\
\hline miR-21-3p agomir & 5'-CAACAGCAGUCGAUGGGCUGU-3' \\
& 3'-CAGCCCAUCGACUGCUGUUGU-5' \\
agomir negative & 5'-UUCUCCGAACGUGUCACGUTT-3' \\
control & 3'-ACGUGACACGUUCGGAGAATT-5' \\
miR-21-3p antagomir & 5'-GACAGCCCAUCGACUGCUGUU-3' \\
antagomir negative & 5'-CAGUACUUUUGUGUAGUACAA-3' \\
control &
\end{tabular}




\section{Cell death detection}

The transferase-mediated deoxyuridine triphosphate-biotin nick end labeling (TUNEL) assay was utilized to evaluate cell death after OGD treatment as we reported previously. ${ }^{20,31}$ Briefly, the cultured cells were collected at $6 \mathrm{~h}$ post-OGD. They were fixed in $4 \%$ paraformaldehyde for $15 \mathrm{~min}$ at room temperature, followed by being treated with $3 \% \mathrm{H}_{2} \mathrm{O}_{2}$ for $30 \mathrm{~min}$ at $37^{\circ} \mathrm{C}$. After being rinsed with PBS, the cells were incubated with the TUNEL reaction mixture (Beyotime, Shanghai, China) and $0.3 \%$ Triton X-100 (Sigma-Aldrich) in a dark room for $2 \mathrm{~h}$ at $37^{\circ} \mathrm{C}$. Immunofluorescent staining for CD31 was then performed. For quantitative measurement, five randomly selected $200 \times$ fields from each section were digitized using a 3-CCD color video camera (Sony DXC-970MD, Tokyo, Japan) with an immunofluorescence microscope (Olympus IX81, Tokyo, Japan). The double immunopositive cells were counted and quantified by the number in each square millimeter. In addition, the expression levels of apoptotic factors, including Bcl-2, cleaved Caspase- 9 , and Caspase- 3 were detected by Western blotting at $6 \mathrm{~h}$ post-OGD.

\section{Enzyme-linked immunosorbent assay (ELISA) assay for inflammatory mediators}

To evaluate the inflammatory response in OGD-treated cells, the culture medium was gathered at $6 \mathrm{~h}$ post-OGD and treatment. The expression levels of inflammatory mediators, including tumor necrosis factor (TNF-) $\alpha$, interleukin (IL)- $1 \beta$, IL-6, and IL-10, were detected referring to the manufacture's instructions (MTA00B, MLB00C, M6000B, M1000B; R\&D, Minneapolis, MN).

\section{Luciferase reporter assay}

To verify whether miR-21-3p directly targeted methionine adenosyltransferase 2B (MAT2B) mRNA in bEnd.3 cells, the luciferase reporter assay was performed. ${ }^{32}$ Luciferase reporter constructs were made by ligating MAT2B 3'UTR fragments containing the predicted binding sites (TargetScan, http://www.targetscan.org/ cgi-bin/targetscan/vert_71/view_gene.cgi?rs=ENST00000518095.1 $\&$ taxid $=10090 \&$ members $=$ miR-21-3p\&showenc $=1 \&$ shownc $=1 \&$ shownc_nc=1\&showncf $1=1 \&$ showncf $2=1 \&$ subset=1\#miR-21-3p; miRDB, http://mirdb.org/cgi-bin/target_detail.cgi?targetID=862670) into luciferase reporter vector, pGL3. Briefly, the fragment was amplified by PCR from mice genomic DNA. The pGL3-MAT2B3'UTR construct was then inserted into the pGL3 control vector containing SV40 promoter (Promega, Madison, WI), using XbaI enzyme. Besides, the mutant type (Mut) of luciferase reporter was generated from the wild type (WT) of luciferase reporter by deleting the binding site for miR-21-3p.

For reporter assay, the bEnd.3 cells were cultured in 96-well plates. The WT or Mut MAT2B-3'UTR was co-transfected with 200pmol miR-21-3p agomir or agomir negative control (sequences listed in Table 1; GenePharma) in neurons using lipofectamine-3000. After $48 \mathrm{~h}$ of incubation, the cells were harvested, and the luciferase activity was measured using a dual-luciferase reporter system (Promega, Madison, WI) referring to the manufacture's instructions.

\section{Short hairpin RNA (ShRNA) vector transfection}

To determine the regulative effect of miR-21-3p on cellular apoptosis and inflammation through targeting MAT2B, a shRNA vector (GenePharma) that can silence the gene expression of MAT2B was transfected into bEnd.3 cells following the manufacturer's instructions. After that, miR-21-3p antagomir transfection and OGD treatment were performed, and the expression levels of MAT2B, Bcl-2, cleaved caspase-9, and nuclear factor kappa B $(\mathrm{NF}-\kappa \mathrm{B})$, were detected by Western blotting at $6 \mathrm{~h}$ post-OGD.

\section{In vivo treatment with miR-21-3p oligomers}

The CCI mice were divided randomly into five groups: miR-21-3p antagomir (21-3p-), antagomir negative control (3p- NC), miR-21$3 p$ agomir $(21-3 p+)$, agomir negative control ( $3 p+N C)$, and injury control (CCI). As reported, the miRNA oligomers were diluted to a final concentration of $50 \mu \mathrm{M}$. Then $2.0-\mu \mathrm{L}$ miRNA oligomers were combined with 5.0- $\mu \mathrm{L}$ lipofectamine-3000 in an RNase-free PCR tube and incubated for $20 \mathrm{~min}$ at room temperature.

Intracerebroventricular infusion was administered $24 \mathrm{~h}$ before CCI modeling. The Hamilton brain infusion syringe was injected stereotaxically into the right lateral ventricle through a burr hole (coordinates: $0.6 \mathrm{~mm}$ caudal to bregma; $1.1 \mathrm{~mm}$ lateral to midline; $2.5 \mathrm{~mm}$ deep from the surface of the skull). Continuous infusion was maintained at the rate of $1.0 \mu \mathrm{L} / \mathrm{min}$. The needle was withdrawn 5 min after the completion of the infusion. The speed of inserting and withdrawing the needle was controlled at $1.5 \mathrm{~mm} / \mathrm{min}$.

To detect the effect of miR-21-3p oligomers treatment, quantitative RT-PCR for miR-21-3p level in BMVECs from the injured cerebral cortex was performed at $24 \mathrm{~h}$ post-CCI. The BMVECs were isolated and acquired by multiple centrifugation and filtration as described above.

\section{Evans Blue (EB) extravasation assay}

EB solution has a high affinity for the albumin in blood circulation, which gives rise to a high-molecular complex $(68.5 \mathrm{kDa})$ with a limited penetration for the intact BBB. For the extravasation assay, $2 \%$ EB solution (Sigma-Aldrich) was injected into the mice through the caudal vein at a dose of $20 \mathrm{mg} / \mathrm{kg}$ at $24 \mathrm{~h}$ post-CCI. After $4 \mathrm{~h}$, the mice were sacrificed by transcardiac perfusion with physiologic saline. As we reported previously, ${ }^{21}$ the injured hemisphere was then dissected, weighed, and incubated in N, N-Dimethylformamide at $37^{\circ} \mathrm{C}$ immediately. After $72 \mathrm{~h}$, the tissue was centrifuged at $1000 \mathrm{~g}$ for $15 \mathrm{~min}$. The supernatant was isolated and detected by a spectrophotometer at the wavelength of $632 \mathrm{~nm}$. According to the standard curve, the quantity of extravasated EB was measured.

\section{In vivo tight junction proteins detection}

The BMVECs from injured cerebral cortex were isolated and acquired at $24 \mathrm{~h}$ post-CCI. Western blotting for occludin, claudin-5 and ZO-1 was performed to quantify the impact of miR-21-3p oligomers treatment on the expression of tight junction proteins after TBI.

\section{Neurological outcome evaluation}

All neurofunctional tests were performed in the behavioral testing room. A video-tracking system (Ethovision 3.0, Noldus Information Technology, Wageningen, Netherlands) was utilized to record the whole procedure of animal activities in training and experimental sessions, including the following tests:

(1) modified Neurological Severity Score (mNSS) test: the mNSS test includes motor, sensory, reflex, and balance assessment. ${ }^{33}$ It was performed at $1,3,7$, and 14 days post-CCI to study the effect of miRNA oligomers treatment on neurological outcome.

(2) Morris Water Maze (MWM) test: the MWM test was performed to evaluate cognitive outcome as we reported previously. ${ }^{19}$ For the spatial acquisition trial, the mice were placed in a pool ( $105 \mathrm{~cm}$ diameter) filled with room temperature water and allowed up to $90 \mathrm{sec}$ to locate a submerged platform. The mice performed four trials a day with a 30-min intertrial interval for four consecutive days (14-17 days). They were introduced in varying quadrants (northwest, northeast, southwest, and southeast) of the pool for each trial, but the location of the platform was fixed. The latency-time to reach 
the platform was recorded, and the four trials were averaged. The probe trial was conducted on day 18 ( $24 \mathrm{~h}$ after the last spatial acquisition trial). In this test, the platform was removed and the mice were allowed to swim freely for $60 \mathrm{sec}$. The percent of the time spent in the goal quadrant was measured.

(3) Novel object recognition test: the test was performed on day 14 post-CCI as reported. ${ }^{34}$ Briefly, the mice were allowed to explore freely a $50 \times 50 \times 50 \mathrm{~cm}$ open-field box (CleverSys, Reston, VA) for $10 \mathrm{~min}$ before experimental sessions. During the first session (familiarization session), they were allowed to explore freely two similar objects. During the second session (test session), one of the two objects was replaced by a novel object. The amount of time that the mice spent on exploring each object was recorded, and the index of exploring time on the novel object over the total exploring time was finally calculated.

\section{Quantitative RT-PCR}

Total RNA was extracted from the microvessels isolated from injured hemispheres of brain tissue or cultured cells using TRIzol reagent (ThermoFisher Scientific). The RNA concentration and quality were evaluated by Nanodrop Spectrophotometer (ND-2000, ThermoFisher Scientific). Reverse transcription and qRT-PCR were performed using the Hairpin-it ${ }^{\mathrm{TM}} \mathrm{miR}-21-3 \mathrm{p} / \mathrm{mRNA}$ RT-PCR Quantitation kit (GenePharma) with corresponding primers (Table 2). All PCR reactions were performed using standard PCR conditions. U6 served as the internal control for miR-21-3p, and glyceraldehyde 3-phosphate dehydrogenase (GAPDH) was used as the internal control for MAT2A and MAT2B. The cycle threshold (Ct value) was detected by a CFX Connect ${ }^{\mathrm{TM}} \mathrm{RT}$-PCR system (Bio-Rad). The data were analyzed using the $2^{-\Delta \Delta C t}$ formula.

\section{Western blotting}

The sodium dodecyl sulfate polyacrylamide gel electrophoresis (SDS/PAGE) and immunoblotting were performed at $6 \mathrm{~h}$ postOGD. The $8 \%$ SDS-acrylamide gel was used for detecting ZO-1. The $10 \%$ SDS-acrylamide gel was used for detecting occludin, Bcl$2, \mathrm{NF}-\kappa \mathrm{B}, \mathrm{I} \kappa \mathrm{B} \alpha, \mathrm{MAT} 2 \mathrm{~A}$, and MAT2B. The $12 \%$ SDS-acrylamide gel was used for detecting claudin-5, cleaved caspase-3, and cleaved caspase-9. GAPDH was used as the internal control. For densitometry, the ChemiDoc ${ }^{\mathrm{TM}}$ XRS+ Imaging System (Bio-Rad, Hercules, CA) was employed. Mean pixel density of each band was detected by the Quantity One software (Bio-Rad). Information about the antibodies used in the study is listed in Table 3.

Table 2. Primer Sequences of miRnAs and mRNAs FOR QUANTITATIVE RT-PCR

\begin{tabular}{|c|c|c|}
\hline Gene Name & Primer & Sequences \\
\hline \multirow[t]{2}{*}{ miR-21-3p } & Forward & $\begin{array}{l}\text { 5'-ACTCTAGAGTCGACACCACTG } \\
\text { ACTCTGATC-3" }\end{array}$ \\
\hline & Reverse & $\begin{array}{l}\text { 5'-ACTCTAGACATGACACAGCTA } \\
\text { CACAACC-3" }\end{array}$ \\
\hline \multirow[t]{2}{*}{ U6 } & Forward & 5'-CTCGCTTCGGCAGCACA-3' \\
\hline & Reverse & 5'-AACGCTTCACGAATTTGCGT-3' \\
\hline \multirow[t]{2}{*}{ MAT2A } & Forward & 5'-ACCCTATGCATGGTTTCAGC-3' \\
\hline & Reverse & 5'-ACCCTGGGAGGAGCTATTG-3' \\
\hline \multirow[t]{2}{*}{ MAT2B } & Forward & 5'-AGGGAACCTTTCACTGGTCTG-3' \\
\hline & Reverse & $5^{\prime}$-ATTTGGAGCAATCGAGCTGAG-3 \\
\hline \multirow[t]{2}{*}{ GAPDH } & Forward & 5'-GCCAAGGCTGTGGGCAAGGT-3' \\
\hline & Reverse & $5^{\prime}$-TCTCCAGGCGGCACGCAGA-3' \\
\hline
\end{tabular}

TABle 3. List of Antibodies

\begin{tabular}{llclr}
\hline Antibody & Manufacturer & Catalogue No. & Application & Dilution \\
\hline Bcl-2 & CST & 3498 & WB & $1: 1000$ \\
CD31 & Abcam & Ab24590 & IF & $1: 200$ \\
cleaved & CST & 9664 & WB & $1: 1000$ \\
$\begin{array}{l}\text { Caspase-3 } \\
\text { cleaved }\end{array}$ & CST & 9509 & WB & $1: 1000$ \\
$\quad$ Caspase-9 & & & & \\
Occuldin & Abcam & ab216327 & WB & $1: 1000$ \\
Claudin-5 & Abcam & ab15106 & WB & $1: 500$ \\
GAPDH & CST & 2118 & WB & $1: 1000$ \\
I $\kappa$ B $\alpha$ & CST & 4814 & WB & $1: 1000$ \\
MAT2A & Abcam & ab77471 & WB & $1: 1000$ \\
MAT2B & Abcam & ab109484 & WB & $1: 1000$ \\
NF- $\kappa$ B & CST & 8242 & WB & $1: 1000$ \\
ZO- 1 & Abcam & ab216880 & WB & $1: 500$ \\
\hline
\end{tabular}

IF, immunofluorescence staining; WB, Western blotting.

\section{Statistical analysis}

All data are based on at least three independent experiments. The data are expressed as mean \pm standard deviation (SD), except for that of the spatial acquisition trials of the MWM test, which is expressed as mean \pm standard error of the mean (SEM). Data of the mNSS test was analyzed using repeated measures analysis of variance (ANOVA) followed by least significant difference (LSD) post hoc analysis. For other data, statistical comparisons were analyzed using one-way ANOVA followed by LSD post hoc analysis or Student $t$ test. A $p$ value $<0.05$ was considered significant.

\section{Results}

\section{Expression level of miR-21-3p was increased in BMVECs of CCI mice and in bEnd. 3 cells after OGD treatment}

BMVECs are the major cellular component of microvascular endothelial barrier, which is an essential part of the highly specialized BBB. They protect the brain from pathogenic microorganisms and other high molecular weight substances in the blood circulation and maintain BBB functioning well. ${ }^{35}$ Thus, we detected the level change on miR-21-3p in BMVECs from the injured cerebral cortex of CCI mice (Fig. 1A) at several time points (from $3 \mathrm{~h}$ to 7 days) after injury using quantitative RT-PCR. We found that CCI led to an increase on the miR-21-3p level soon after injury. It was increased since $3 \mathrm{~h}$ post-CCI, reached the peak at $24 \mathrm{~h}$ post$\mathrm{CCI}$, and gradually decreased to the baseline over $72 \mathrm{~h}$ (Fig. 1B). These findings indicated that miR-21-3p might be an important factor that regulates BBB damage in CCI mice.

The bEnd. 3 cells are a commercially available mouse BBB cell line. They were cultured to explore further the function and mechanism of miR-21-3p on BBB damage in vitro. We performed immunofluorescence staining for endothelial cell marker CD31 after the cells grew to confluent (Fig. 1C). Most of the cells were positively immunostained, suggesting that the cultured cells are pure endothelial cells (Fig. 1D). As reported previously, ${ }^{36}$ the OGD-treated bEnd.3 cells injury model is applicable to in vitro research for traumatic BBB damage. We administered OGD treatment on cultured cells and observed that they became blebbed or shrunken (Fig. 1E). In addition, HPICM scanning was performed to observe the microstructure change on tight junctions among bEnd.3 cells soon after OGD treatment. The tight junctions could be observed clearly among 
cultured cells, but disappeared after injury (Fig. 1F, 1G). These results confirmed that OGD treatment destroyed the microvascular endothelial barrier and exerted a similar effect to $\mathrm{CCI}$ that induced in vivo $\mathrm{BBB}$ damage.

Based on this work, we detected the expression level of miR-21$3 p$ in bEnd. 3 cells at several time points (from $1 \mathrm{~h}$ to $48 \mathrm{~h}$ ) after OGD treatment using quantitative RT-PCR. The OGD treatment led to an increase of the miR-21-3p level in bEnd.3 cells. It was soon increased since $1 \mathrm{~h}$ post-OGD, reached the peak at $6 \mathrm{~h}$ postOGD, and gradually decreased to the baseline over $24 \mathrm{~h}$ (Fig. 1H). This variation trend of miR-21-3p expression in bEnd.3 cells after OGD treatment was quite similar to that in BMVECs from injured cerebral cortex after CCI, suggesting that miR-21-3p may also play a significant role in the pathological development of the in-vitro BBB injury model.

A
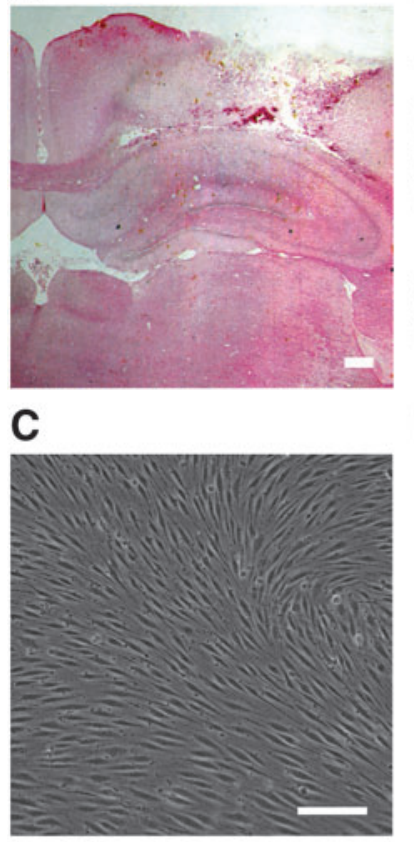

E
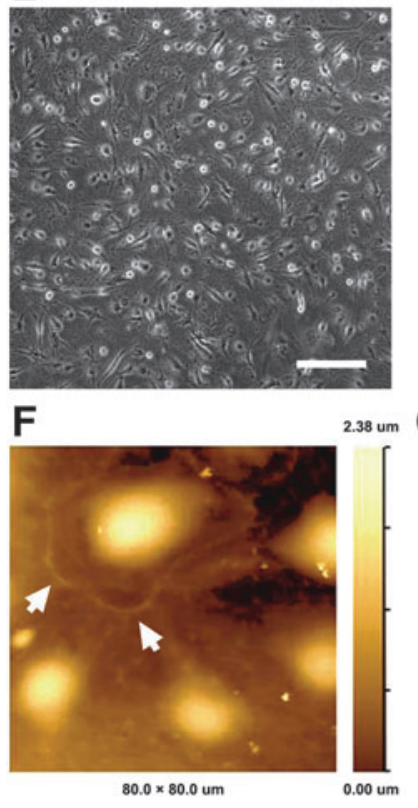

B
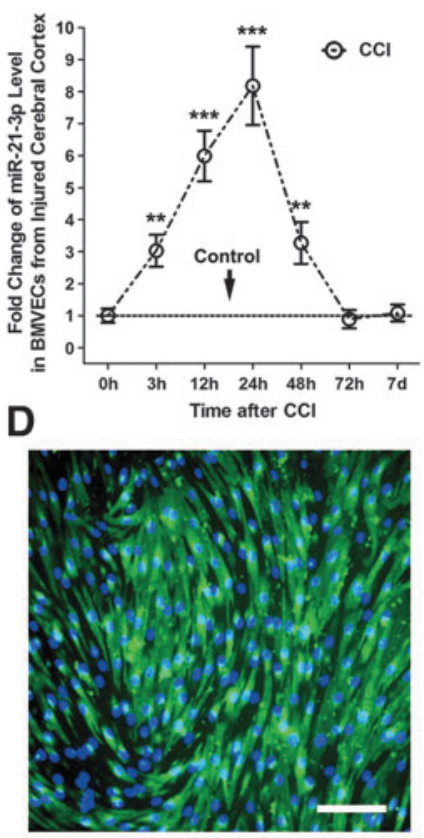

H

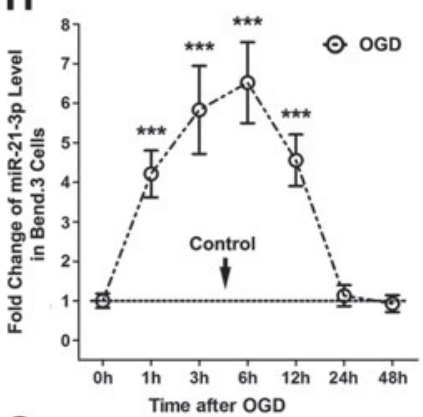

G

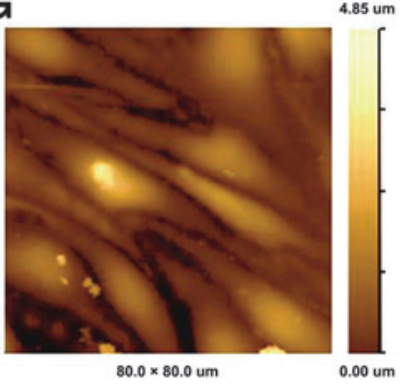

\section{Downregulation on miR-21-3p level could alleviate $O G D$-induced $B B B$ damage}

We downregulated the miR-21-3p level in bEnd.3 cells by transfecting miR-21-3p antagomir and evaluated the transfection efficiency using FAM-labeled miR-21-3p antagomir. The immunostaining showed that $88.5 \%$ of bEnd.3 cells were transfected successfully (Fig. 2A). In addition, we quantified miR-21-3p level in cultured cells after the transfection using quantitative RT-PCR at $6 \mathrm{~h}$ post-OGD, when miR-21-3p level reached the peak in the OGD group. It suggested that the miR-21-3p level could be downregulated significantly by miR-21-3p antagomir (Fig. 2B).

The permeability of OGD-treated BBB was measured using HRP flux assay. We found that miR-21-3p antagomir alleviated the leakage of HRP from $6+1 \mathrm{~h}$ to $6+4 \mathrm{~h}$ post-OGD (Fig. 2C). Further, tight junction proteins, such as occludin, claudin 5, and ZO-1, are the components that connect BMVECs, which sealed the intracellular gaps among BMVECs and maintain the integrity of BBB. ${ }^{37}$ We quantified the expression levels of occludin, claudin-5, and ZO1 in Bend. 3 cells at $6 \mathrm{~h}$ post-OGD by Western blotting. It indicated that their levels were decreased at $6 \mathrm{~h}$ post-OGD and could be upregulated by miR-21-3p antagomir (Fig. 2D, 2E). Taken together, downregulation on miR-21-3p levels could alleviate OGDinduced BBB damage. Thus, the increased miR-21-3p level in bEnd. 3 cells after OGD treatment, as well as that in BMVECs after TBI, was bad for restoration of injured BBB.

\section{Downregulation on miR-21-3p level could inhibit cellular apoptosis after OGD treatment}

The impact of the miR-21-3p level on cell death after OGD treatment was evaluated by TUNEL assay. We observed a large number of double-positively labeled cells (TUNEL and CD31) at

FIG. 1. The temporal profile of miR-21-3p level in brain microvascular endothelial cells (BMVECs) of controlled cortical impact (CCI) mice and bEnd.3 cells after oxygen glucose deprivation/reoxygenation (OGD) treatment. (A) The hematoxylin and eosin staining of injured mouse brain at $24 \mathrm{~h}$ post-CCI. Scale bar $=250 \mu \mathrm{m}$. (B) The altered miR-21-3p level in BMVECs of CCI mice from $0 \mathrm{~h}$ to 7 days post-injury. CCI led to an increase on miR-21-3p level in BMVECs soon after injury. It was increased since $3 \mathrm{~h}$ post-CCI, reached the peak at $24 \mathrm{~h}$ post-CCI, and gradually decreased to the baseline over $72 \mathrm{~h}$. (C) Cultured bEnd.3 cells under the transmission light microscope. Scale bar $=50 \mu \mathrm{m}$. (D) Immunofluorescence staining of CD31 for cell identification, which revealed pure culture of bEnd. 3 cells. Scale bar $=50 \mu \mathrm{m}$. (E) OGD-treated bEnd.3 cells under the transmission light microscope. Note that blebbed (spherical and bulky) and shrunken cells were observed widely. Scale bar $=$ $50 \mu \mathrm{m}$. (F, G) Continuous hopping probe ion conductance microscopy topological scanning on tight junctions (white arrow) among bEnd. 3 cells before and after OGD treatment. Note that the structure of tight junctions could not be observed in OGD-treated cells. Scale bar $=50 \mu \mathrm{m}$. (H) The altered miR-21-3p level in bEnd.3 cells from $0 \mathrm{~h}$ to $48 \mathrm{~h}$ post-OGD. The OGD treatment led to an increase of miR$21-3 p$ level in bEnd. 3 cells. It was soon increased since $1 \mathrm{~h}$ postOGD, reached the peak at $6 \mathrm{~h}$ post-OGD, and gradually decreased to the baseline over $24 \mathrm{~h}$. The quantitative reverse transcription polymerase chain reaction data were analyzed using the $2^{-\Delta \Delta C t}$ method, in which the miR-21-3p level of uninjured mice and cells (presented as the dotted line) were used as controls. $n=6 /$ group. Data represent the mean \pm standard deviation of at least $\mathrm{N}=3$ independent experiments. $* * p<0.01, * * * p<0.001$. Color image is available online. 
A B

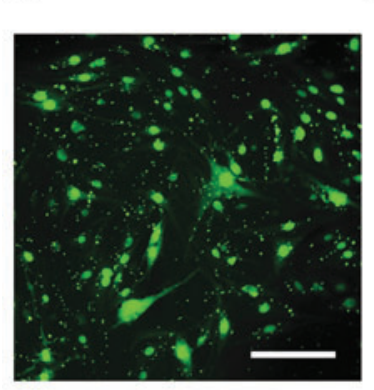

B

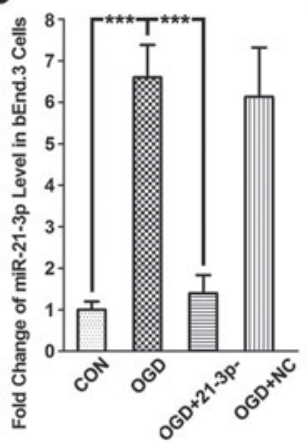

C

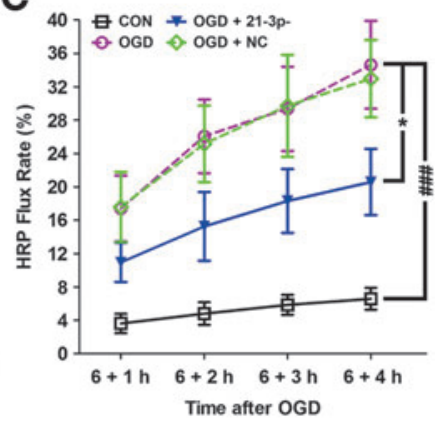

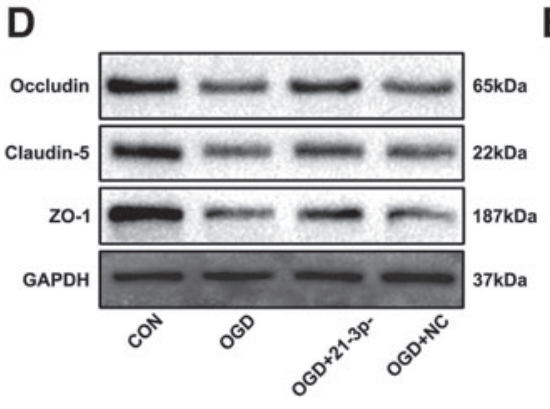

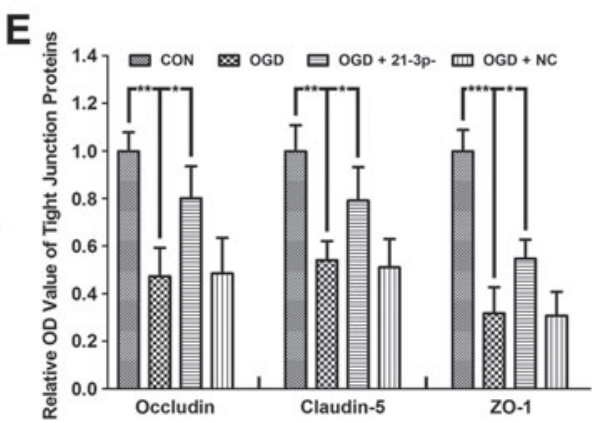

FIG. 2. The impact of miR-21-3p on permeability of in vitro established blood-brain barrier (BBB) after oxygen glucose deprivation/ reoxygenation (OGD) treatment. (A) Immunostaining of bEnd.3 cells transfecting with FAM-labeled miR-21-3p antagomir. Note that almost all cells were transfected successfully. Scale bar $=50 \mu \mathrm{m}$. (B) The altered miR-21-3p level in bEnd.3 cells after miR-21-3p oligomers transfection at $6 \mathrm{~h}$ post-OGD. Note that the miR-21-3p level was significantly downregulated by miR-21-3p antagomir. (C) The horseradish peroxidase (HRP) flux rate from $6+1 \mathrm{~h}$ to $6+4 \mathrm{~h}$ post-OGD. Note that miR-21-3p antagomir alleviated the leakage of HRP at the four time points. (D) The immunoblot and (E) quantitative data of occludin, claudin-5m and ZO-1 in bEnd.3 cells at $6 \mathrm{~h}$ post-OGD. Note that OGD treatment induced loss of tight junction protein, and miR-21-3p antagomir could alleviate the impact. Thus, increased miR-21-3p level in bEnd. 3 cells was bad for restoration of injured BBB after OGD treatment. $n=6 /$ group. Data represent the mean \pm standard deviation of at least $\mathrm{N}=3$ independent experiments. ${ }^{*} p<0.05,{ }^{*} p<0.01, * * * p<0.001$. \#\#\#p $<0.001$. Color image is available online.

$6 \mathrm{~h}$ post-OGD, suggesting that OGD treatment could lead to remarkable bEnd. 3 cell death. In addition, the number of doubleimmunoreactive cells was decreased in the OGD +21-3p- group, compared with the OGD group. It indicated that miR-21-3p antagomir suppressed OGD-induced cell death (Fig. 3A, 3B).

The expression levels of apoptotic factors (Bcl-2, cleaved caspase-9, and cleaved caspase-3) were quantified at $6 \mathrm{~h}$ post-OGD by Western Blotting. We found that miR-21-3p antagomir promoted the expression of Bcl-2 and inhibited the expression of cleaved caspase-9 and cleaved caspase-3 (Fig. 3C, 3D). Therefore, downregulation on miR-21-3p level could inhibit cellular apoptosis after OGD treatment.

\section{Downregulation of the miR-21-3p level could suppress inflammatory response after OGD treatment}

To demonstrate the impact of miR-21-3p on the inflammatory response in bEnd. 3 cells after OGD treatment, we quantified the expression levels of inflammatory cytokines (TNF- $\alpha$, IL-1 $\beta$, IL-6, and IL-10) in culture medium at $6 \mathrm{~h}$ post-OGD using ELISA assay. The OGD treatment induced inflammation in bEnd. 3 cells, characterized by increased expression on proinflammatory cytokines (TNF- $\alpha$, IL- $1 \beta$ and IL-6) and decreased expression on antiinflammatory cytokines (IL-10). miR-21-3p antagomir could reverse the expression changes on inflammatory mediators. It inhibited the expression of TNF- $\alpha$, IL- $1 \beta$, and IL- 6 and promoted the expression of IL-10, thus exerting an anti-inflammatory effect in the injured cells (Fig. 4A).
$\mathrm{NF}-\kappa \mathrm{B}$ signaling plays a central role in the development of the inflammatory response, which can regulate the expression of large amounts of downstream inflammatory mediators. ${ }^{38}$ In unstimulated cells, it is sequestered in the cytoplasm by $\mathrm{I} \kappa \mathrm{B}$ inhibitory proteins through blocking the nuclear localization signals. On cellular stimulation by immune and proinflammatory responses, NF- $\kappa \mathrm{B}$-activating agents can induce the phosphorylation of $\mathrm{I} \kappa \mathrm{B} \alpha$ and target it for rapid degradation that releases NF- $\kappa \mathrm{B}$ to the nucleus, where it regulates downstream gene expression. ${ }^{39}$ Thus, we detected the expression levels of NF- $\kappa \mathrm{B}$ and $\mathrm{I} \kappa \mathrm{B} \alpha$ in Bend. 3 cells at $6 \mathrm{~h}$ post-OGD by Western blotting. We found that miR-21-3p antagomir inhibited the expression of NF- $\kappa \mathrm{B}$ and promoted that of $\mathrm{I} \kappa \mathrm{B} \alpha$ (Fig. $4 \mathrm{~B}, 4 \mathrm{C}$ ), which suggested that downregulation on miR-21-3p level could suppress NF- $\kappa$ B controlled inflammatory response after OGD treatment.

\section{miR-21-3p regulated cellular apoptosis and inflammation through targeting MAT2B}

Methionine adenosyltransferase is the cellular enzyme that catalyzes the synthesis of S-adenosylmethionine, the principal biological methyl donor and regulator of hepatocyte proliferation, death, and differentiation. In previous research on hepatocellular carcinoma, MAT2A and MAT2B have been reported to be targeted by miR-21-3p and inhibit cellular apoptosis under its regulation. ${ }^{40}$ They have also been confirmed to interact with NF- $\kappa$ B. ${ }^{41,42}$ In addition, MAT2A and MAT2B are both expressed widely in the brain, including the cerebral cortex and hippocampus (https://www .proteinatlas.org/). 

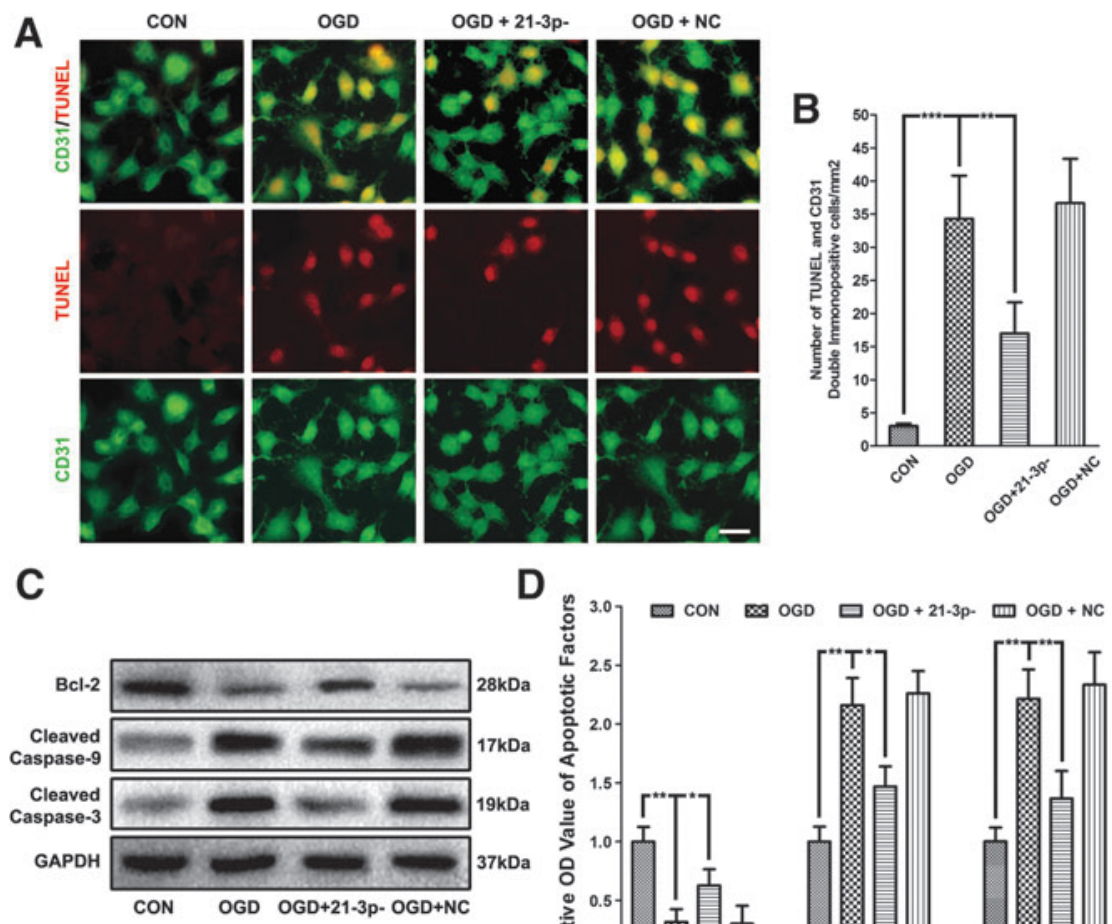

FIG. 3. The impact of miR-21-3p on cellular apoptosis after oxygen glucose deprivation/reoxygenation (OGD) treatment. (A) The immunostaining and (B) quantitative data of dead cells at $6 \mathrm{~h}$ post-OGD detected by transferase-mediated deoxyuridine triphosphatebiotin nick end labeling (TUNEL) assay. Note that the number of double-immunoreactive (TUNEL and CD31) cells was reduced by miR-21-3p antagomir, suggesting that it suppressed OGD-induced bEnd.3 cell death. Scale bar $=100 \mu \mathrm{m}$. (C) The immunoblot and (D) quantitative data of apoptotic factors (Bcl-2, cleaved caspase-9, and cleaved caspase-3) in bEnd.3 cells at $6 \mathrm{~h}$ post-OGD. Note that miR21-3p antagomir promoted the expression of Bcl-2, and inhibited the expression of cleaved caspase-9 and cleaved caspase-3, suggesting that it inhibited cellular apoptosis after OGD treatment. $n=6$ /group. Data represent the mean \pm standard deviation of at least $\mathrm{N}=3$ independent experiments. ${ }^{*} p<0.05, * * p<0.01$. Color image is available online.

To study whether miR-21-3p could target MAT2A and MAT2B in bEnd. 3 cells, Western blotting and quantitative RT-PCR were performed to assess the protein and mRNA levels of MAT2A and MAT2B at $6 \mathrm{~h}$ post-OGD. We found that miR-21-3p antagomir promoted the expression of MAT2B, but had no influence on the expression of MAT2A on both protein and mRNA levels (Fig. 5AC). Thus, MAT2A was excluded from the potential target of miR21-3p in bEnd. 3 cells. Subsequently, we searched for the potential binding site in miR-21-3p for MAT2B 3'UTR using miRDB (Fig. 5D) and conducted the luciferase reporter assay after cotransfecting bEnd.3 cells with MAT2B 3'UTR constructs, which contained the putative miR-21-3p binding site with either miR-21$3 p$ agomir or scrambled oligonucleotides (agomir negative control). The miR-21-3p inhibited the luciferase activity of the WT, but not the Mut 3'UTR reporter construct (Fig. 5E), suggesting that miR-21-3p could target directly MAT2B and downregulate its expression by binding to the 3'UTR sites.

To determine further the regulative effect of miR-21-3p on cellular apoptosis and inflammation through targeting MAT2B, we transfected a shRNA vector that can silence the expression of MAT2B in bEnd.3 cells (MAT2B-). We found that silencing MAT2B could block the suppressive effect of miR-21-3p antagomir on cellular apoptosis and inflammation in bEnd. 3 cells after OGD treatment. Compared with the OGD +21-3p- group, a decreased expression on $\mathrm{Bcl}-2$, and an increased expression on cleaved caspase- 9 and NF- $\kappa \mathrm{B}$, could be observed in the MAT2B- group at
$6 \mathrm{~h}$ post-OGD (Fig. 5F, 5G). These results indicated that miR-21-3p regulated cellular apoptosis and inflammation mainly through directly targeting MAT2B.

\section{miR-21-3p antagomir treatment could alleviate $B B B$ damage after TBI}

To confirm further the protective effect of miR-21-3p antagomir on in vivo BBB damage after TBI, we manipulated the miR-21-3p level in the injured brain of CCI mice by intracerebroventricular infusion of miR-21-3p oligomers. We found that the miR-21-3p level in BMVECs from injured cerebral cortex was downregulated (or upregulated) after miR-21-3p antagomir (or agomir) treatment at $24 \mathrm{~h}$ post-CCI, when the miR-21-3p level reached the peak in the CCI group. In addition, no difference in miR-21-3p levels was observed among the CCI group, 3p- NC group, and 3p+ NC group (Fig. 6A).

The permeability of BBB after TBI was evaluated at $24 \mathrm{~h}$ post$\mathrm{CCI}$ using EB extravasatoin assay. As shown in Figures $6 \mathrm{~B}$ and $6 \mathrm{C}$, the extravasation of EB solution from injured hemisphere after TBI could be alleviated (or aggravated) by miR-21-3p antagomir (or agomir). The expression levels of tight junction proteins has proved to be decreased after TBI in our previous research. ${ }^{21} \mathrm{We}$ quantified the expression of occludin, claudin-5, and $\mathrm{ZO}-1$ at $24 \mathrm{~h}$ post-CCI using Western blotting and found that their expressions in injured cerebral cortex were all increased (or decreased) after miR-21-3p antagomir (or agomir) treatment (Fig. 6D, 6E). It indicated that 

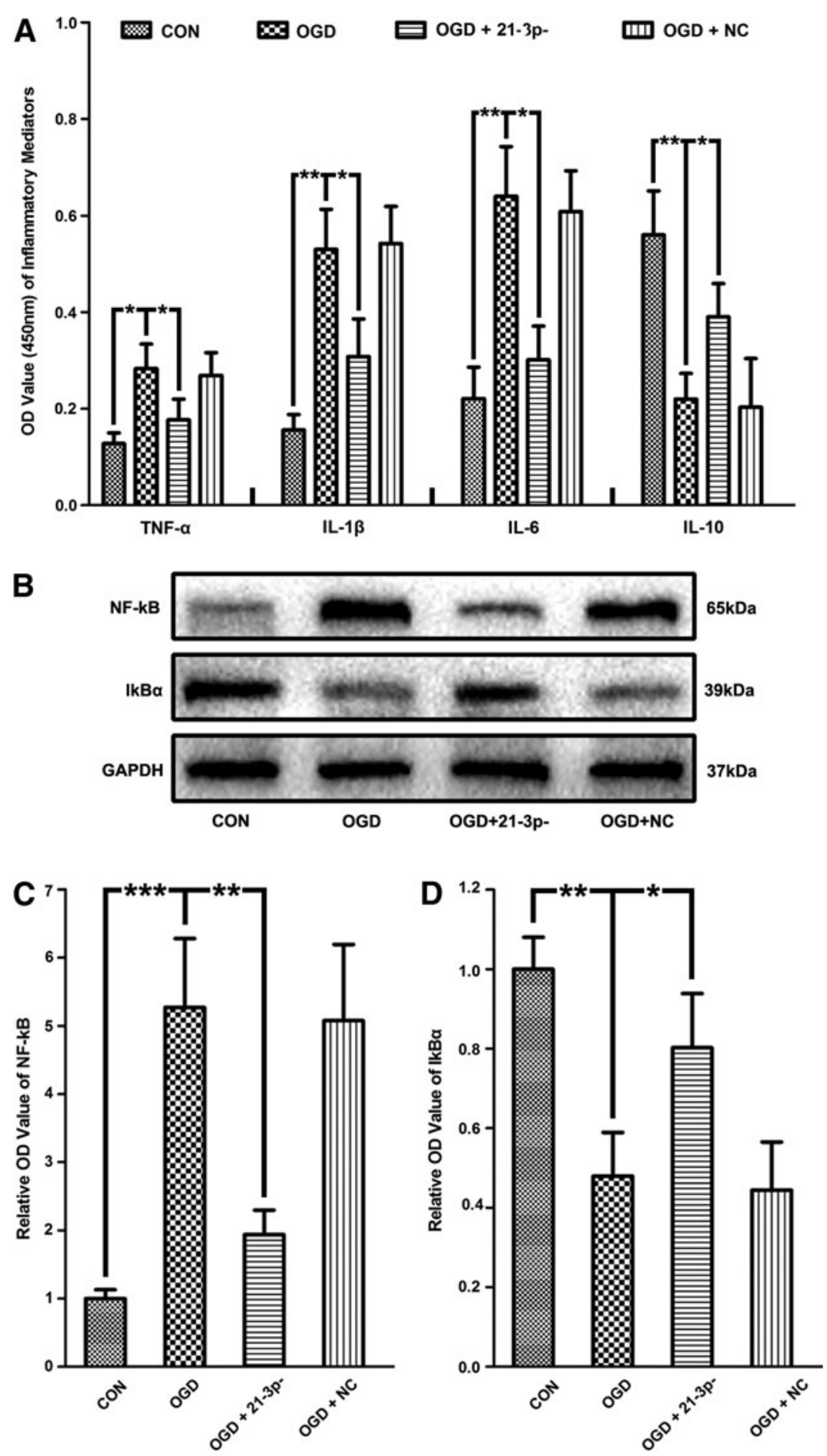

FIG. 4. The impact of miR-21-3p on inflammatory response after oxygen glucose deprivation/reoxygenation (OGD) treatment. (A) The expression levels of inflammatory mediators (tumor necrosis factor [TNF]- $\alpha$, interleukin [IL]-1 $\beta$, IL-6, and IL-10) in culture medium at $6 \mathrm{~h}$ post-OGD detected by enzyme-linked immunosorbent assay. Note that the OGD treatment induced inflammation in bEnd. 3 cells, characterized by increased expression on proinflammatory cytokines (TNF- $\alpha$, IL-1 $\beta$, and IL-6) and decreased expression on anti-inflammatory cytokines (IL-10). miR-21-3p antagomir could reverse the expression changes on inflammatory mediators, thus exerting an anti-inflammatory effect in injured cells. (B) The immunoblot and (C) quantitative data of nuclear factor (NF)- $\kappa \mathrm{B}$ signaling factors $(N F-\kappa \mathrm{B}$ and $\mathrm{I} \kappa \mathrm{B} \alpha)$ at $6 \mathrm{~h}$ post-OGD. Note that miR-21-3p antagomir inhibited the expression of NF- $\kappa \mathrm{B}$, and promoted the expression of $\mathrm{I} \kappa \mathrm{B} \alpha$, suggesting that it suppressed the $\mathrm{NF}-\kappa \mathrm{B}$ controlled inflammatory response. $n=6 /$ group. Data represent the mean \pm standard deviation of at least $\mathrm{N}=3$ independent experiments. $* p<0.05, * * p<0.01, * * * p<0.001$. 
A

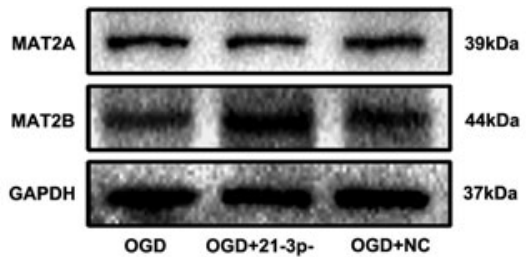

D

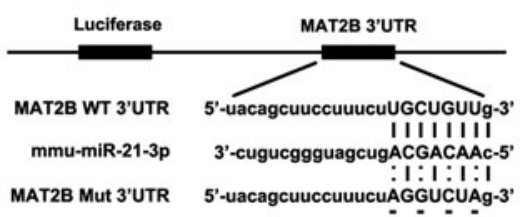

B

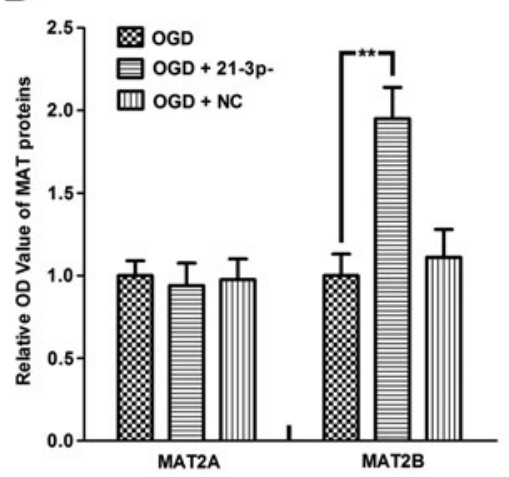

C

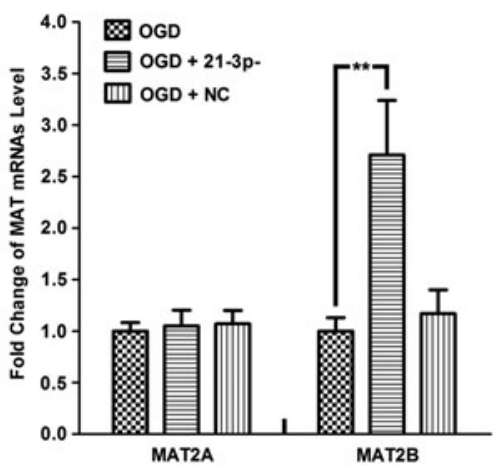

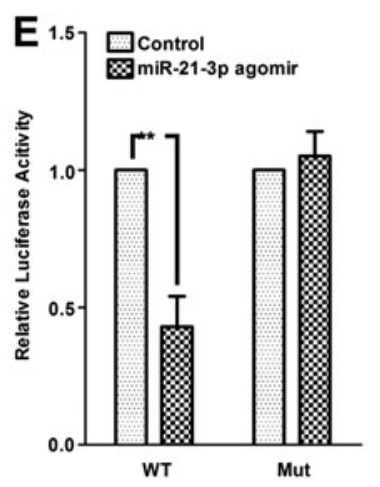
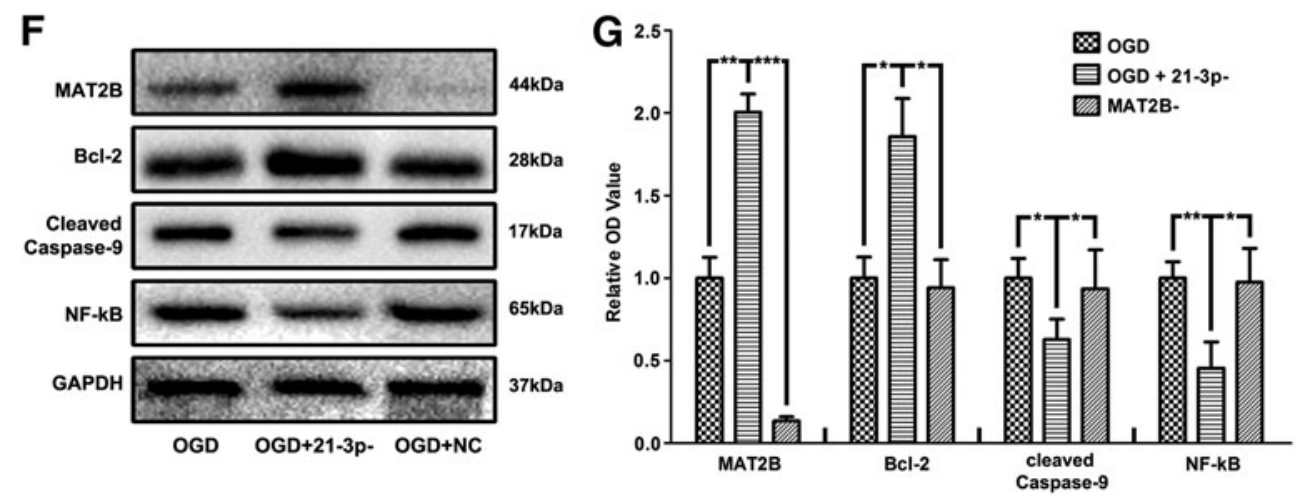

FIG. 5. miR-21-3p regulated cellular apoptosis and inflammation through targeting MAT2B. (A) The immunoblot of MAT2A and MAT2B in bEnd.3 cells after at $6 \mathrm{~h}$ post- oxygen glucose deprivation/reoxygenation (OGD). The quantitative data of (B) protein and (C) messenger ribonucleic acid (mRNA) levels of MAT2A and MAT2B in bEnd.3 cells at $24 \mathrm{~h}$ post-OGD. Note that miR-21-3p antagomir promoted the expression of MAT2B, but had no influence on the expression of MAT2A on both protein and mRNA levels. (D) Schematic representation of the potential binding sites for miR-21-3p in the MAT2B 3'UTR. Seed sequences of the wild type (MAT2B WT 3'UTR) and mutant type (MAT2B Mut 3'UTR) luciferase reporter were shown in the binding site. (E) The relative luciferase activity of the WT and Mut reporter constructs, which were co-transfected with either miR-21-3p agomir or agomir negative control. Data are presented as the ratio of luciferase activity from the agomir negative control versus miR-21-3p agomir transfected bEnd.3 cells. Note that miR-21-3p inhibited the luciferase activity of the WT, but not the Mut 3'UTR reporter construct, suggesting that miR-21-3p could directly target MAT2B and downregulate its expression by binding to the 3'UTR sites. (F) The immunoblot and (G) quantitative data of expression levels of MAT2B, Bcl-2, cleaved caspase- 9 , and NF- $\kappa \mathrm{B}$ were detected in three groups of bEnd. 3 cells at $6 \mathrm{~h}$ postOGD: OGD treatment (OGD), miR-21-3p antagomir transfection with OGD treatment (OGD +21-3p-), and MAT2B silenced bEnd.3 cells with miR-21-3p antagomir transfection and OGD treatment (MAT2B-). Compared with the OGD +21-3p- group, the MAT2Bgroup showed a decreased expression on Bcl- 2 and an increased expression on cleaved caspase- 9 and NF- $\kappa \mathrm{B}$, suggesting that silencing MAT2B expression could block the suppressive effect of miR-21-3p antagomir on cellular apoptosis and inflammation in bEnd.3 cells after OGD treatment. Therefore, miR-21-3p regulated cellular apoptosis and inflammation mainly through directly targeting MAT2B. $n=6$ /group. Data represent the mean \pm standard deviation of at least $\mathrm{N}=3$ independent experiments. ${ }^{*} p<0.05, * * p<0.01, * * * p<0.001$.

miR-21-3p antagomir reduced TBI-induced loss of tight junction proteins. Therefore, miR-21-3p antagomir treatment could alleviate BBB damage after TBI.

\section{miR-21-3p antagomir treatment could improve the neurological outcome after TBI}

The neurological function of CCI mice was evaluated by the mNSS test, the MWM test, and novel object recognition test. In the mNSS test, lower score demonstrates better neurological function. As shown in Figure 7A, no difference on the neurological score was observed among all groups at day 1 post-CCI. The recovery of neurological function began at 3 days and lasted to 14 days postCCI, when mice still had residual neurological deficiencies on sensory and beam balance. In addition, compared with the CCI group, the neurological score was decreased in the 21-3p- group
$(\mathrm{F}=6.06, p=0.0265)$, but increased in the $21-3 \mathrm{p}+$ group $(\mathrm{F}=8.05$, $p=0.014)$, suggesting that downregulation on miR-21-3p level in the brain improved the neurological score of CCI mice.

In the MWM test, the spatial acquisition trial was performed from 14 days to 17 days post-CCI to test spatial learning ability. Escape latency, which represents the capability to navigate from a start location to a submerged platform, was decreased gradually in the testing procedure, indicating that a spatial memory was established (repeated-measures ANOVA, F $(3,105)=813.89, p<0.001)$. The probe trial was conducted at 18 days post-CCI to test the retrograde reference memory, in which more time spent in the goal quadrant demonstrates better memory. We found that the brain injury led to an obvious increase on escape latency and a decrease on time spent in the goal quadrant of CCI mice. Compared with the CCI group, the 21-3p- group displayed a decrease on the escape latency and an increase on the time spent in the goal quadrant, whereas reversed 

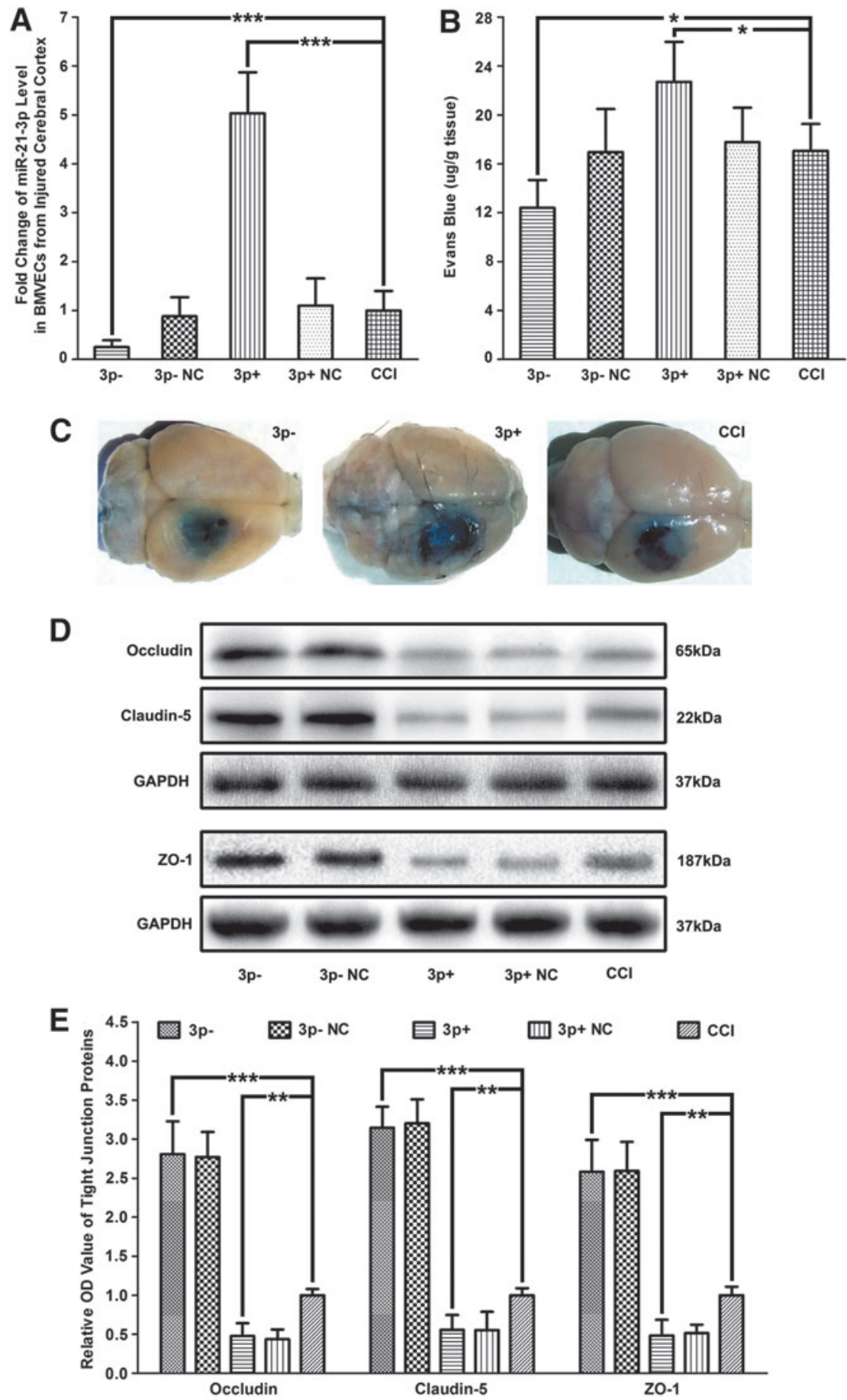

FIG. 6. The impact of miR-21-3p oligomers treatment on blood-brain barrier (BBB) damage after traumatic brain injury (TBI). (A) The altered miR-21-3p level in brain microvascular endothelial cells (BMVECs) from injured cerebral cortex at $24 \mathrm{~h}$ post-controlled cortical impact (CCI) after miR-21-3p oligomers treatment. Note that the miR-21-3p level could be upregulated (or downregulated) by intracerebroventricular infusion of miR-21-3p agomir (or antagomir). The quantitative reverse transcription polymerase chain reaction data were analyzed using the $2^{-\Delta \Delta \mathrm{Ct}}$ method, in which miR-21-3p level of injury control group (presented as the dotted line) were used as controls. (B) The quantitative data and (C) general view of EB extravasted from injured hemisphere at $24 \mathrm{~h}$ post-CCI. Note that the leakage of Evans Blue (EB) solution after TBI was alleviated (or aggravated) by miR-21-3p antagomir (or agomir). (D) The immnuoblot and (E) quantitative data of tight junction proteins (occludin, claudin-5, and ZO-1) in BMVECs from lesioned cerebral cortex at $24 \mathrm{~h}$ post-CCI. Note that the expression of tight junction proteins in the injured brain was promoted (or inhibited) by miR-21-3p antagomir (or agomir). Thus, miR-21-3p antagomir treatment could alleviate BBB damage after TBI. Scale bar $=500 \mu \mathrm{m}$. $n=7-9 /$ group. Data represent the mean \pm standard deviation of at least $\mathrm{N}=3$ independent experiments. ${ }^{*} p<0.05, * * p<0.01, * * * p<0.001$. Color image is available online. 
results were observed in the $21-3 p+$ group (Fig. 7B, 7C). In addition, there was no difference on the swim speed among all groups, suggesting that the different performance was not because of motor impairments. Thus, these results indicated that downregulation on miR-21-3p level in the brain improved the spatial learning ability of CCI mice.

In the object recognition test, the amount of time taken to explore the new object provides an indicator for recognitive memory. We found that the index of exploring time on the novel object over the total exploring time was increased (or decreased) after miR-21-3p antagomir (or agomir) treatment (Fig. 7D). Therefore, downregulation of miR-21-3p level in the brain improved the recognitive memory of CCI mice. Taken together, our data of the three behavioral tests indicated that miR-21-3p antagomir treatment could improve the neurological outcome after TBI.

\section{Discussion}

miRNAs have been studied widely in the etiology of a variety of diseases, including cancer, cardiovascular diseases, and neurological diseases. Compared with these chronic diseases, the research on miRNAs in acute diseases started much later. As to TBI, the related research began to be reported since 2009. Redell and coworkers ${ }^{43}$ and our group ${ }^{18}$ first detected the miRNAs expression changes in the injured hippocampus and cerebral cortex of TBI rats, respectively, and both identified the increased expression of miR21-5p after TBI using miRNA microarray, quantitative RT-PCR, and in-situ hybridization. These results were confirmed further by Miao and associates ${ }^{44}$ using improved seventh generation of miRCURYTM LNA array.

Based on the findings, miR-21-5p has become the hotspot in miRNA-related TBI studies. Redell and colleagues ${ }^{45}$ predicted PDCD4 and Tiam1 to be the target genes of miR-21-5p using a combination of miRanda, TargetScan, and PicTar algorithms, suggesting that miR-21-5p may be involved in regulating apoptosis and hippocampal synaptic plasticity in the injured brain. ${ }^{46}$ Our group found that miR-21-5p is expressed mainly in neurons, gliacytes, and BMVECs in the brain, and its levels in these cells are all increased in response to TBI.

Upregulation of the miR-21-5p level in the injured brain by intracerebroventricular infusion of miR-21-5p agomir confers a better neurological outcome after TBI. The protective effects of miR-21-5p are exerted by: (1) inhibiting neuronal apoptosis and promoting angiogenesis through targeting PTEN and activating
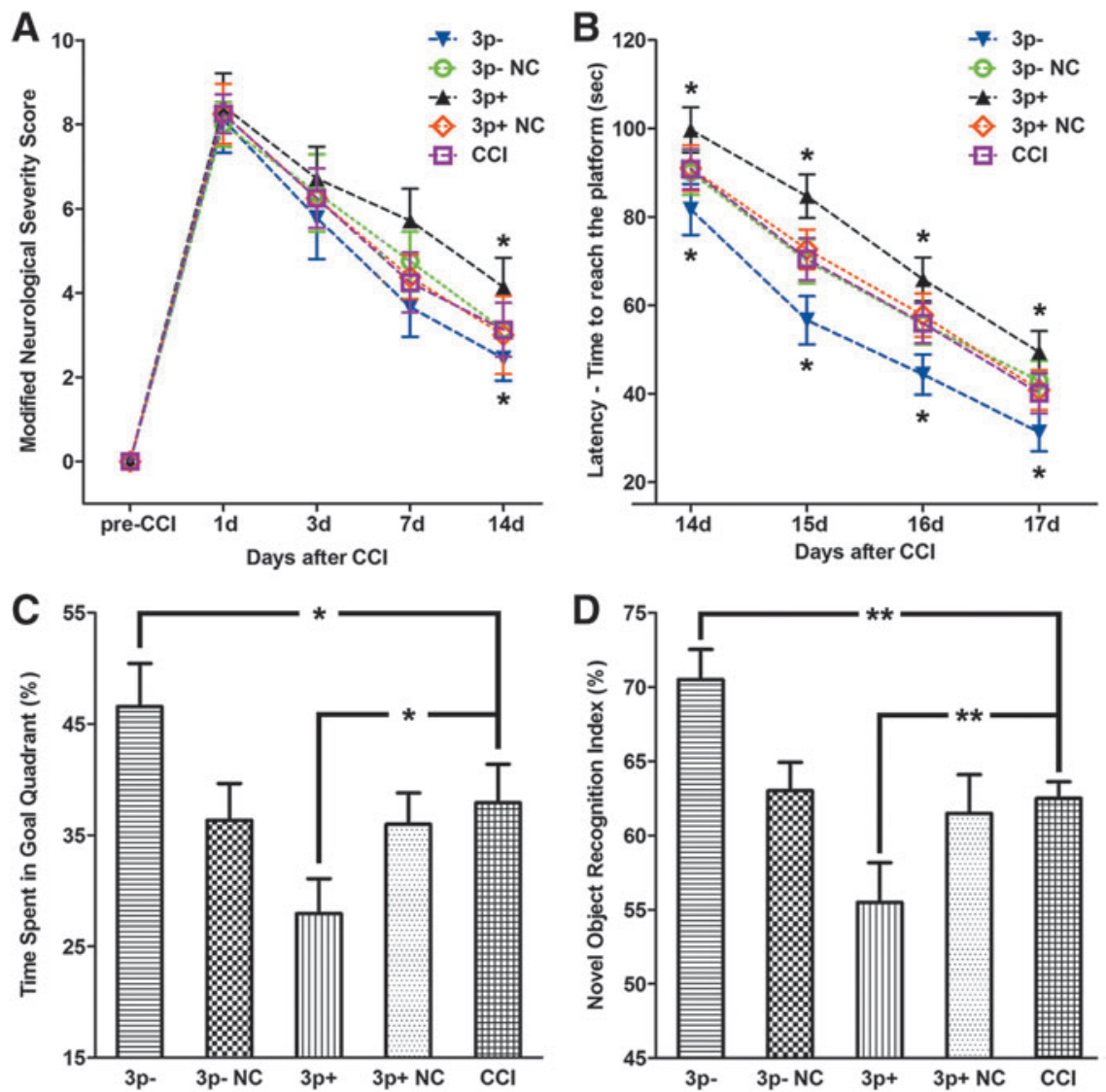

FIG. 7. The impact of miR-21-3p oligomers treatment on the neurological outcome after traumatic brain injury (TBI). The neurological function of controlled cortical impact (CCI) mice was evaluated by (A) modified Neurological Severity Score test, (B, C) Morris Water Maze test, and (D) novel object recognition test. (A) miR-21-3p antagomir (or agomir) treatment decreased (or increased) the neurological score on $14 \mathrm{~d}$ post-CCI. (B, C) Escape latency for the spatial acquisition trial was shortened, and time spent in the goal quadrant for the probe trial was improved by miR-21-3p antagomir treatment. By contrast, miR-21-3p agomir treatment exerted a reversed effect. (D) The index of exploring time on the novel object over the total exploring time was increased (or decreased) by miR21-3p antagomir (or agomir) treatment. Taken together, miR-21-3p antagomir treatment could improve the neurological outcome of CCI mice. $n=7-9$ /group. Data represent the mean \pm standard deviation $(\mathrm{A}, \mathrm{C}, \mathrm{D})$ or mean \pm standard error of the mean $(\mathrm{B})$ of at least $\mathrm{N}=3$ independent experiments. ${ }^{*} p<0.05, * * p<0.01$. Color image is available online. 
Akt signaling ${ }^{19,20}$; (2) alleviating BBB damage by promoting the expression of tight junction proteins via activating the Angiopoietin1/Tie-2 axis in BMVECs. ${ }^{21-23}$ In addition, Sandhir and colleagues ${ }^{47}$ suggested that there is no change of the miR-21-5p level after TBI in aged mice, and the expression of its predicted target genes, such as PTEN and PDCD4, are promoted. Because the activation of PTEN and PDCD4 promote cellular apoptosis, the diminished miR-21-5p injury response may contribute to a poor prognosis in the aging brain.

miR-21-3p is another mature miRNA derived from pre-miR-21 after Dicer Procession other than miR-21-5p. As a potential diagnostic biomarker, especially for a variety of tumors, miR-21-3p has aroused great interest for research in recent years. Its specific roles, such as regulating apoptosis and inflammation, were not the same or even opposite in different diseases. As to TBI, previous research reported that the miR-21-3p level in the injured brain was increased at $1 \mathrm{~h}, 6 \mathrm{~h}$, and $12 \mathrm{~h}$ post-injury. ${ }^{27}$ Its function and underlying mechanism in TBI, however, especially on BBB damage, have not been clarified until now.

In this research, we first performed the CCI mouse model and detected the temporal profile of miR-21-3p level in BMVECs after injury. Its level was soon increased since $3 \mathrm{~h}$ post-CCI and lasted until $72 \mathrm{~h}$ post-CCI. For in vitro research, we cultured bEnd. 3 cells, a mouse BBB cell line and performed the OGD-treated cell injury model. A similar change- that the miR-21-3p level was increased from $1 \mathrm{~h}$ to $24 \mathrm{~h}$ post-OGD—was also observed. Therefore, we conclude that the impact of miR-21-3p on BMVECs is exerted in the early stage after injury.

The impact of miR-21-3p on BBB damage was detected at the time points when the miR-21-3p level reached the peak in bEnd.3 cells after OGD treatment and in BMVECs from the injured brain of CCI mice. We found that increased miR-21-3p in BMVECs after injury was bad for restoration of BBB, characterized by increased BBB leakage and decreased expression of tight junction proteins.

Subsequently, we studied the mechanism of miR-21-3p in regulating BBB damage from two aspects: apoptosis and inflammation. Traumatic BBB leakage induces apoptosis in the injured brain, which in turn exacerbates BBB damage. miR-21-3p has been proven to promote cellular apoptosis in hepatoma. ${ }^{40}$ From this, we hypothesized that miR-21-3p may exert a proapoptosis effect in BMVECs after TBI. The results of in vitro experiments confirmed our hypothesis, suggesting that downregulation of the miR-21-3p level could promote the expression of antiapoptotic factors (Bcl-2) and inhibit the expression of proapoptotic factors (cleaved caspase9 and cleaved caspase-3), thus suppress apoptosis in injured cells.

Neural inflammation after TBI is an important pathological change induced by BBB damage. It is represented by microglial and astrocytic activation, infiltration of peripheral inflammatory cells, as well as the release of inflammatory cytokines including TNF- $\alpha$, IL- $1 \beta$, IL- 6 and IL- $10 .{ }^{48}$ As reported previously, miR-21$3 p$ could promote inflammation in various diseases. ${ }^{26,49,50} \mathrm{We}$ hypothesized that miR-21-3p induced BMVECs inflammation after TBI. Thus, we detected its impact on levels of inflammatory cytokines in injured bEnd. 3 cells and found that miR-21-3p antagomir could inhibit the expression of pro-nflammatory cytokines (TNF- $\alpha$, IL- $1 \beta$ and IL-6) and promoted the expression of anti-inflammatory cytokines (IL-10). Meanwhile, as the upstream inducer of the inflammatory cascade, NF- $\kappa \mathrm{B}$ signaling was also suppressed. Taken together, it could be inferred that the anti-inflammatory effect of miR-21-3p antagomir in injured BBB is exerted via inhibiting NF- $\kappa$ B signaling.

MAT2A and MAT2B are the subtypes of MAT that catalyzes the synthesis of S-adenosylmethionine and function as important regulators in the development of hepatoma. Because they have been proven to be targeted by miR-21-3p in hepatocytes ${ }^{40}$ and are both expressed widely in the brain, we tried to verify whether they were targeted by miR-21-3p in bEnd.3 cells. The results indicated that miR-21-3p could inhibit the expression of MAT2B and regulate cellular apoptosis and inflammation mainly through targeting it. Other previous reports also support these findings with circumstantial evidence. Specifically, MAT2B can alleviate apoptosis in various tumor cells. ${ }^{51-53}$ In addition, overexpression of MAT2B is associated with the inhibition of $\mathrm{mTOR},{ }^{54}$ which is a classic upstream promoter of NF- $\kappa \mathrm{B}$ signaling, ${ }^{55,56}$ suggesting that MAT2B may alleviate cellular apoptosis and NF- $\kappa \mathrm{B}$ signaling controlled inflammatory response. Consequently, our research fully identified that miR-21-3p could aggravate BBB damage after TBI by promoting cellular apoptosis and inflammation through targeting MAT2B.

$\mathrm{BBB}$ is a structural and functional unit composed mainly of BMVECs, gliacytes, pericytes, neurons, and extracellular matrix. Thus, increased miR-21-3p in BMVECS after TBI may also exert indirect influence on other cells in the neurovascular unit. Specifically, the proapoptosis effect of miR-21-3p in BMVECs could exacerbate neural death in surrounding brain tissue for spreading of apoptotic factors via exocrine secretion or exosomes transportation. ${ }^{57}$ In addition, miR-21-3p contributes to inflammation in BMVECs after TBI. It may lead to the infiltration of plasma molecules (including inflammatory mediators) and circulating cells (such as peripheral inflammatory cells) into the brain, which finally result in aggravated neural inflammation and brain damage ${ }^{58}$ From this, miR-21-3p could also exert a significant impact on the whole neurovascular unit through regulating BBB leakage after TBI.

For our future research, other potential target genes and downstream signaling pathways of miR-21-3p will be studied to further clarify its mechanism in regulating BBB damage. Bioinformatics analysis, including target prediction, GO analysis, KEGG analysis, and combined genetic interaction network analysis are being performed. In addition, the roles of miR-21-3p in other cells in the brain, such as neurons and microglias, after TBI are also planned to be elucidated. The coregulative effect of miR-21-5p and miR-21-3p on BBB damage in different TBI stages will be studied by simultaneously manipulating their levels in experimental animals and cultured cells. Nevertheless, this study explains the roles of miR$21-3 p$ in regulating the pathological development of traumatic BBB damage, thus expanding the previous understanding of the function and mechanism of miR-21 in TBI.

\section{Conclusion}

The major discovery of our research is that miR-21-3p level in BMVECs from injured cerebral cortex of CCI mice and bEnd.3 cells with OGD treatment are both increased significantly after injury. Increased miR-21-3p in BMVECs after TBI is bad for restoration of injured BBB. The miR-21-3p antagomir treatment, which downregulated miR-21-3p expression in BMVECs, could alleviate BBB damage by suppressing cellular apoptosis and NF$\kappa \mathrm{B}$ controlled inflammatory response, thus improving the neurological outcome of CCI mice. These functions of miR-21-3p are exerted by directly targeting MAT2B. Therefore, miR-21-3p could be a potential therapeutic target for intervention in TBI treatment. Downregulation on its level in the injured brain is a promising therapeutic strategy for traumatic BBB damage.

\section{Acknowledgments}

This research was supported by grants from the National Natural Science Foundation of China (grant no. 81501055, 81772060, 
81720108015 and 81471252), Tianjin Traditional Medicine Key Research Project (grant no. 2018001) and Tianjin Medical University General Hospital Youth Cultivation Foundation (grant no. ZYYFY2016007). The authors appreciate Chunsheng Kang, Ying Li, Li Liu, Weiyun Cui and Lei Zhou from Tianjin Neurological Institute for their technical support.

\section{Author Disclosure Statement}

No competing financial interests exist.

\section{References}

1. Maas, A.I., Menon, D.K., Adelson, P.D., Andelic, N., Bell, M.J., Belli, A., Bragge, P., Brazinova, A., Buki, A., Chesnut, R.M., Citerio, G., Coburn, M., Cooper, D.J., Crowder, A.T., Czeiter, E., Czosnyka, M., Diaz-Arrastia, R., Dreier, J.P., Duhaime, A.C., Ercole, A., van Essen, T. A., Feigin, V.L., Gao, G., Giacino, J., Gonzalez-Lara, L.E., Gruen, R. L., Gupta, D., Hartings, J. A., Hill, S., Jiang, J.Y., Ketharanathan, N., Kompanje, E.J., Lanyon, L., Laureys, S., Lecky, F., Levin, H., Lingsma, H.F., Maegele, M., Majdan, M., Manley, G., Marsteller, J., Mascia, L., McFadyen, C., Mondello, S., Newcombe, V., Palotie, A., Parizel, P.M., Peul, W., Piercy, J., Polinder, S., Puybasset, L., Rasmussen, T. E., Rossaint, R., Smielewski, P., Soderberg, J., Stanworth, S.J., Stein, M. B., von Steinbuchel, N., Stewart, W., Steyerberg, E.W., Stocchetti, N., Synnot, A., Te Ao, B., Tenovuo, O., Theadom, A., Tibboel, D., Videtta, W., Wang, K K., Williams, W.H., Wilson, L., and Yaffe, K. (2017). Traumatic brain injury: integrated approaches to improve prevention, clinical care, and research. Lancet Neurol. 16, 987-1048.

2. Feigin, V L., Theadom, A., Barker-Collo, S., Starkey, N.J., McPherson, K., Kahan, M., Dowell, A., Brown, P., Parag, V., Kydd, R., Jones, K., Jones, A., and Ameratunga, S. (2013). Incidence of traumatic brain injury in New Zealand: a population-based study. Lancet Neurol. 12, 53-64.

3. Levin, H., Smith, D. (2013). Traumatic brain injury: networks and neuropathology. Lancet Neurol. 12, 15-16.

4. Shlosberg, D., Benifla, M., Kaufer, D., and Friedman, A. (2010). Blood-brain barrier breakdown as a therapeutic target in traumatic brain injury. Nat. Rev. Neurol. 6, 393-403.

5. Edwards, P., Arango, M., Balica, L., Cottingham, R., El-Sayed, H., Farrell, B., Fernandes, J., Gogichaisvili, T., Golden, N., Hartzenberg, B., Husain, M., Ulloa, M.I., Jerbi, Z., Khamis, H., Komolafe, E., Laloe, V., Lomas, G., Ludwig, S., Mazairac, G., Munoz Sanchez Mde, L., Nasi, L., Olldashi, F., Plunkett, P., Roberts, I., Sandercock, P., Shakur, H., Soler, C., Stocker, R., Svoboda, P., Trenkler, S., Venkataramana, N.K., Wasserberg, J., Yates, D., and Yutthakasemsunt, S. (2005). Final results of MRC CRASH, a randomised placebocontrolled trial of intravenous corticosteroid in adults with head injury—outcomes at 6 months. Lancet 365, 1957-1959.

6. Chen, X., Zhao, Z., Chai, Y., Luo, L., Jiang, R., Dong, J., and Zhang, J. (2013). Stress-dose hydrocortisone reduces critical illness-related corticosteroid insufficiency associated with severe traumatic brain injury in rats. Crit. Care 17, R241.

7. Chen, X., Zhao, Z., Chai, Y., Luo, L., Jiang, R., and Zhang, J. (2014). The incidence of critical-illness-related-corticosteroid-insufficiency is associated with severity of traumatic brain injury in adult rats. J. Neurol. Sci. 342, 93-100.

8. Jiang, J.Y., Lyeth, B.G., Kapasi, M.Z., Jenkins, L.W., and Povlishock, J.T. (1992). Moderate hypothermia reduces blood-brain barrier disruption following traumatic brain injury in the rat. Acta Neuropathol. $84,495-500$.

9. Thal, S.C., and Neuhaus, W. (2014). The blood-brain barrier as a target in traumatic brain injury treatment. Arch. Med. Res. 45, 698-710.

10. Dietrich, W.D., and Bramlett, H.M. (2016). Therapeutic hypothermia and targeted temperature management in traumatic brain injury: clinical challenges for successful translation. Brain Res. 1640, 94-103.

11. Menge, T., Zhao, Y., Zhao, J., Wataha, K., Gerber, M., Zhang, J., Letourneau, P., Redell, J., Shen, L., Wang, J., Peng, Z., Xue, H., Kozar, R., Cox, C.S., Jr., Khakoo, A.Y., Holcomb, J.B., Dash, P.K., and Pati, S. (2012). Mesenchymal stem cells regulate blood-brain barrier integrity through TIMP3 release after traumatic brain injury. Sci. Transl. Med. 4, 161ra150.
12. Walker, P.A., Shah, S.K., Jimenez, F., Gerber, M.H., Xue, H., Cutrone, R., Hamilton, J.A., Mays, R.W., Deans, R., Pati, S., Dash, P.K., and Cox, C.S., Jr. (2010). Intravenous multipotent adult progenitor cell therapy for traumatic brain injury: preserving the blood brain barrier via an interaction with splenocytes. Exp. Neurol. 225, 341-352.

13. Huang, X.T., Zhang, Y.Q., Li, S.J., Li, S.H., Tang, Q., Wang, Z.T., Dong, J.F., and Zhang, J.N. (2013). Intracerebroventricular transplantation of ex vivo expanded endothelial colony-forming cells restores blood-brain barrier integrity and promotes angiogenesis of mice with traumatic brain injury. J. Neurotrauma 30, 2080-2088.

14. Nasser, M., Bejjani, F., Raad, M., Abou-El-Hassan, H., Mantash, S., Nokkari, A., Ramadan, N., Kassem, N., Mondello, S., Hamade, E., Darwish, H., Zibara, K., and Kobeissy, F. (2016). Traumatic brain injury and blood-brain barrier cross-talk. CNS Neurol. Disord. Drug Targets 15, 1030-1044.

15. Skolnick, B.E., Maas, A.I., Narayan, R.K., van der Hoop, R.G., MacAllister, T., Ward, J.D., Nelson, N.R., and Stocchetti, N. (2014). A clinical trial of progesterone for severe traumatic brain injury. N. Engl. J. Med. 371, 2467-2476.

16. Robertson, C.S., Hannay, H.J., Yamal, J.M., Gopinath, S., Goodman, J.C., Tilley, B.C., Baldwin, A., Rivera Lara, L., Saucedo-Crespo, H., Ahmed, O., Sadasivan, S., Ponce, L., Cruz-Navarro, J., Shahin, H., Aisiku, I.P., Doshi, P., Valadka, A., Neipert, L., Waguspack, J.M., Rubin, M.L., Benoit, J.S., and Swank, P. (2014). Effect of erythropoietin and transfusion threshold on neurological recovery after traumatic brain injury: a randomized clinical trial. JAMA 312, 36-47.

17. Kumar, A., and Loane, D.J. (2012). Neuroinflammation after traumatic brain injury: opportunities for therapeutic intervention. Brain Behav. Immun. 26, 1191-1201.

18. Lei, P., Li, Y., Chen, X., Yang, S., and Zhang, J. (2009). Microarray based analysis of microRNA expression in rat cerebral cortex after traumatic brain injury. Brain Res. 1284, 191-201.

19. Ge, X.T., Lei, P., Wang, H.C., Zhang, A.L., Han, Z.L., Chen, X., Li, S.H., Jiang, R.C., Kang, C.S., and Zhang, J.N. (2014). miR-21 improves the neurological outcome after traumatic brain injury in rats. Sci. Rep. 4, 6718.

20. Han, Z., Chen, F., Ge, X., Tan, J., Lei, P., and Zhang, J. (2014). miR21 alleviated apoptosis of cortical neurons through promoting PTENAkt signaling pathway in vitro after experimental traumatic brain injury. Brain Res. 1582, 12-20.

21. Ge, X., Han, Z., Chen, F., Wang, H., Zhang, B., Jiang, R., Lei, P., and Zhang, J. (2015). MiR-21 alleviates secondary blood-brain barrier damage after traumatic brain injury in rats. Brain Res. 1603, 150-157.

22. Ge, X., Huang, S., Gao, H., Han, Z., Chen, F., Zhang, S., Wang, Z., Kang, C., Jiang, R., Yue, S., Lei, P., and Zhang, J. (2016). miR-21-5p alleviates leakage of injured brain microvascular endothelial barrier in vitro through suppressing inflammation and apoptosis. Brain Res. $1650,31-40$.

23. Ge, X., Lei, P., and Zhang, J. (2015). miR-21, a potential therapeutic target of alleviating blood-brain barrier damage after traumatic brain injury. RNA Dis. 2, e642.

24. Yan, M., Chen, C., Gong, W., Yin, Z., Zhou, L., Chaugai, S., and Wang, D.W. (2015). miR-21-3p regulates cardiac hypertrophic response by targeting histone deacetylase-8. Cardiovasc. Res. 105, 340-352.

25. Baez-Vega, P.M., Echevarria Vargas, I.M., Valiyeva, F., EncarnacionRosado, J., Roman, A., Flores, J., Marcos-Martinez, M.J., and VivasMejia, P.E. (2016). Targeting miR-21-3p inhibits proliferation and invasion of ovarian cancer cells. Oncotarget 7, 36321-36337.

26. Gordillo, G.M., Biswas, A., Khanna, S., Pan, X., Sinha, M., Roy, S., and Sen, C.K. (2014). Dicer knockdown inhibits endothelial cell tumor growth via microRNA 21a-3p targeting of Nox-4. J. Biol. Chem. 289, 9027-9038.

27. Meissner, L., Gallozzi, M., Balbi, M., Schwarzmaier, S., Tiedt, S., Terpolilli, N.A., and Plesnila, N. (2016). Temporal profile of microRNA expression in contused cortex after traumatic brain injury in mice. J. Neurotrauma 33, 713-720.

28. Xu, X., Gao, W., Cheng, S., Yin, D., Li, F., Wu, Y., Sun, D., Zhou, S., Wang, D., Zhang, Y., Jiang, R., Zhang, J. (2017). Anti-inflammatory and immunomodulatory mechanisms of atorvastatin in a murine model of traumatic brain injury. J. Neuroinflammation 14, 167.

29. Zacharek, A., Chen, J., Zhang, C., Cui, X., Roberts, C., Jiang, H., Teng, H., and Chopp, M. (2006). Nitric oxide regulates Angiopoietin1/ Tie2 expression after stroke. Neurosci. Lett. 404, 28-32. 
30. Chen, X., Zhu, H., Liu, X., Lu, H., Li, Y., Wang, J., Liu, H., Zhang, J., Ma, Q., and Zhang, Y. (2013). Characterization of two mammalian cortical collecting duct cell lines with hopping probe ion conductance microscopy. J. Membr. Biol. 246, 7-11.

31. Han, Z., Ge, X., Tan, J., Chen, F., Gao, H., Lei, P., and Zhang, J. (2015). Establishment of lipofection protocol for efficient miR21 transfection into cortical neurons in vitro. DNA Cell. Biol. 34, 703-709.

32. Huang, S., Ge, X., Yu, J., Han, Z., Yin, Z., Li, Y., Chen, F., Wang, H., Zhang, J., and Lei, P. (2018). Increased miR-124-3p in microglial exosomes following traumatic brain injury inhibits neuronal inflammation and contributes to neurite outgrowth via their transfer into neurons. FASEB J. 32, 512-528.

33. Chen, J., Sanberg, P.R., Li, Y., Wang, L., Lu, M., Willing, A.E., Sanchez-Ramos, J., and Chopp, M. (2001). Intravenous administration of human umbilical cord blood reduces behavioral deficits after stroke in rats. Stroke 32, 2682-2688.

34. Leger, M., Quiedeville, A., Bouet, V., Haelewyn, B., Boulouard, M., Schumann-Bard, P., and Freret, T. (2013). Object recognition test in mice. Nat. Protoc. 8, 2531-2537.

35. Ruck, T., Bittner, S., Epping, L., Herrmann, A.M., and Meuth, S.G. (2014). Isolation of primary murine brain microvascular endothelial cells. J. Vis. Exp. 93, e52204.

36. Lin, L., Wang, Q., Qian, K., Cao, Z., Xiao, J., Wang, X., Li, X., and $\mathrm{Yu}, \mathrm{Z}$. (2018). bFGF protects against oxygen glucose deprivation/ reoxygenation-induced endothelial monolayer permeability via S1PR1-dependent mechanisms. Mol. Neurobiol. 55, 3131-3142.

37. Haseloff, R.F., Dithmer, S., Winkler, L., Wolburg, H., and Blasig, I.E. (2015). Transmembrane proteins of the tight junctions at the blood-brain barrier: structural and functional aspects. Semin. Cell Dev. Biol. 38, 16-25.

38. Chen, S., Jiang, S., Zheng, W., Tu, B., Liu, S., Ruan, H., and Fan, C. (2017). RelA/p65 inhibition prevents tendon adhesion by modulating inflammation, cell proliferation, and apoptosis. Cell Death Dis. 8, e2710.

39. Courtois, G., and Gilmore, T.D. (2006). Mutations in the NF-kappaB signaling pathway: implications for human disease. Oncogene 25, 6831-6843.

40. Lo, T.F., Tsai, W.C., and Chen, S.T. (2013). MicroRNA-21-3p, a berberine-induced miRNA, directly down-regulates human methionine adenosyltransferases $2 \mathrm{~A}$ and $2 \mathrm{~B}$ and inhibits hepatoma cell growth. PLoS One 8, e75628.

41. Yang, H., Ara, A.I., Magilnick, N., Xia, M., Ramani, K., Chen, H., Lee, T D., Mato, J.M., and Lu, S.C. (2008). Expression pattern, regulation, and functions of methionine adenosyltransferase 2 beta splicing variants in hepatoma cells. Gastroenterology 134, 281-291.

42. Frau, M., Feo, F., and Pascale, R.M. (2013). Pleiotropic effects of methionine adenosyltransferases deregulation as determinants of liver cancer progression and prognosis. J. Hepatol. 59, 830-841.

43. Redell, J.B., Liu, Y., and Dash, P.K. (2009). Traumatic brain injury alters expression of hippocampal microRNAs: potential regulators of multiple pathophysiological processes. J. Neurosci. Res. 87, 1435-1448.

44. Miao, W., Bao, T.H., Han, J.H., Yin, M., Yan, Y., Wang, W.W., and Zhu, Y.H. (2015). Voluntary exercise prior to traumatic brain injury alters miRNA expression in the injured mouse cerebral cortex. Braz. J. Med. Biol. Res. 48, 433-439.

45. Redell, J.B., Zhao, J., and Dash, P.K. (2011). Altered expression of miRNA-21 and its targets in the hippocampus after traumatic brain injury. J. Neurosci. Res. 89, 212-221.

46. Pan, Y.B., Sun, Z.L., and Feng, D.F. (2017). The role of microRNA in traumatic brain injury. Neuroscience 367, 189-199.

47. Sandhir, R., Gregory, E., and Berman, N.E. (2014). Differential response of miRNA-21 and its targets after traumatic brain injury in aging mice. Neurochem. Int. 78, 117-121.

48. Chiu, C.C., Liao, Y.E., Yang, L.Y., Wang, J.Y., Tweedie, D., Karnati, H.K., Greig, N.H., and Wang, J.Y. (2016). Neuroinflammation in animal models of traumatic brain injury. J. Neurosci. Methods 272, 38-49.

49. Degueurce, G., D’Errico, I., Pich, C., Ibberson, M., Schutz, F., Montagner, A., Sgandurra, M., Mury, L., Jafari, P., Boda, A., Meunier,
J., Rezzonico, R., Brembilla, N. C., Hohl, D., Kolios, A., Hofbauer, G., Xenarios, I., and Michalik, L. (2016). Identification of a novel PPARbeta/delta/miR-21-3p axis in UV-induced skin inflammation. EMBO Mol. Med. 8, 919-936.

50. Cavallini, A., Rotelli, M.T., Lippolis, C., Piscitelli, D., Digennaro, R., Covelli, C., Carella, N., Accetturo, M., and Altomare, D.F. (2017). Human microRNA expression in sporadic and FAP-associated desmoid tumors and correlation with beta-catenin mutations. Oncotarget 8, 41866-41875.

51. Wang, Q., Liu, Q.Y., Liu, Z.S., Qian, Q., Sun, Q., and Pan, D.Y (2008). Lentivirus mediated shRNA interference targeting MAT2B induces growth-inhibition and apoptosis in hepatocelluar carcinoma. World J. Gastroenterol. 14, 4633-4642.

52. Lei, Y., Zhang, B., Zhang, Y., Zhao, Y., Sun, J., Zhang, X., and Yang, S. (2016). Lentivirus-mediated downregulation of MAT2B inhibits cell proliferation and induces apoptosis in melanoma. Int. J. Oncol. 49, 981-990.

53. Yang, H., Zheng, Y., Li, T. W., Peng, H., Fernandez-Ramos, D., Martinez-Chantar, M.L., Rojas, A.L., Mato, J.M., and Lu, S.C. (2013). Methionine adenosyltransferase $2 \mathrm{~B}, \mathrm{HuR}$, and sirtuin 1 protein crosstalk impacts on the effect of resveratrol on apoptosis and growth in liver cancer cells. J. Biol. Chem. 288, 23161-23170.

54. Yang, Y., Li, X., Sun, Q., He, B., Jia, Y., Cai, D., and Zhao, R. (2016). Folate deprivation induces cell cycle arrest at G0/G1 phase and apoptosis in hippocampal neuron cells through down-regulation of IGF-1 signaling pathway. Int. J. Biochem. Cell Biol. 79, 222-230.

55. Rizwan, H., Mohanta, J., Si, S., and Pal, A. (2017). Gold nanoparticles reduce high glucose-induced oxidative-nitrosative stress regulated inflammation and apoptosis via tuberin-mTOR/NF-kappaB pathways in macrophages. Int. J. Nanomedicine 12, 5841-5862.

56. Temiz-Resitoglu, M., Kucukkavruk, S.P., Guden, D.S., Cecen, P., Sari, A.N., Tunctan, B., Gorur, A., Tamer-Gumus, L., Buharalioglu, C.K., Malik, K.U., and Sahan-Firat, S. (2017). Activation of mTOR/ IkappaB-alpha/NF-kappaB pathway contributes to LPS-induced hypotension and inflammation in rats. Eur. J. Pharmacol. 802, 7-19.

57. de Rivero Vaccari, J.P., Brand, F., 3rd, Adamczak, S., Lee, S.W., Perez-Barcena, J., Wang, M.Y., Bullock, M.R., Dietrich, W.D., and Keane, R.W. (2016). Exosome-mediated inflammasome signaling after central nervous system injury. J. Neurochem. 136, Suppl 1, 39-48.

58. Perez-Polo, J.R., Rea, H.C., Johnson, K.M., Parsley, M.A., Unabia, G.C., Xu, G., Infante, S. K., Dewitt, D.S., and Hulsebosch, C.E. (2013). Inflammatory consequences in a rodent model of mild traumatic brain injury. J. Neurotrauma 30, 727-740.

Address correspondence to: Ping Lei, $M D, P h D$

Department of Geriatrics

Tianjin Medical University General Hospital

154 Anshan Road

Tianjin 300052

China

E-mail: leiping1974@163.com

and

Jianning Zhang, $M D, P h D$ Department of Neurosurgery

Tianjin Medical University General Hospital

154 Anshan Road

Tianjin 300052

China

E-mail: jianningzhang@hotmail.com 